\title{
Background Optimization for the Neutron Time-of-Flight Spectrometer NEAT
}

\author{
G. Günther ${ }^{\mathrm{a}}$, M. Russina ${ }^{\mathrm{a}, *}$ \\ ${ }^{a}$ Helmholtz-Zentrum Berlin für Materialien und Energie GmbH, Lise-Meitner-Campus, \\ Hahn-Meitner-Platz 1, 14109 Berlin
}

\section{Abstract}

The neutron time-of-flight spectrometer NEAT at BER II is currently undergoing a major upgrade where an important aspect is the prevention of parasitic scattering to enhance the signal-to-noise ratio. Here, we discuss the impact of shielding to suppress parasitic scattering from two identified sources of background: the sample environment and detector tubes. By means of Monte Carlo simulations and a modification of the analytical model of Copley et al. [Copley and Cook, 1994], the visibility functions of instrument parts are computed for different shielding configurations. According to three selection criteria, namely suppression of background, transmission and detection limit, the parameters of an oscillating radial collimator are optimized for NEAT's default setup. Moreover, different configurations of detector shielding are discussed to prevent crosstalk within the radial detector system.

\section{Outline of the NEAT spectrometer}

8

The direct time-of-flight cold neutron spectrometer NEAT at BER II is developed to study dynamics in the time domain from $10^{-13}-10^{-11} \mathrm{~s}$ and structure at the nanometre scale. Currently, it has almost completed a comprehensive upgrade to maintain its competitive edge among the best in the class of instruments worldwide.

NEAT uses a ballistic neutron guide with supermirror optics to transport the neutrons. The guide starts $1.5 \mathrm{~m}$ behind the cold source and ends in an

${ }^{*}$ Corresponding author, margarita.russina@helmholtz-berlin.de 
exchangeable focusing section $30 \mathrm{~cm}$ or $50 \mathrm{~cm}$ before the sample position, which is $64 \mathrm{~m}$ away from the surface of the cold source. An integrated, about $30 \mathrm{~m}$ long cascade of seven choppers realises well-defined neutron pulses in the range from $2-18 \AA$.

Around the sample, 416 position sensitive ${ }^{3}$ He-detector tubes of $2 \mathrm{~m}$ height and $2.54 \mathrm{~cm}$ diameter are arranged radially at a distance of $3 \mathrm{~m}$. The detectors are composed of 13 structural units, so-called detector modules, comprising 32 detector tubes, in total covering an angle from $-81^{\circ}$ to $143^{\circ}$ in the horizontal direction and about $\pm 18^{\circ}$ vertically.

The end section of the neutron guide as well as the secondary spectrometer are placed in a vacuum chamber whose inner surface is covered with $\mathrm{Cd}$.

\section{Background suppression}

In spectroscopy the signals of interest are generally accompanied by signals that lack the characteristic features of the object under consideration. The latter usually differ in origin and are subsumed as noise. With regard to neutrons as the incident radiation energy, a large amount of noise stems from parts of the instrument where neutrons scatter inadvertently or their absorption process emits gamma rays that trigger detector readouts [1]. Consequently, it is crucial to eliminate sources of spurious scattering since the background can hide the fine (e.g. inelastic) features of a signal.

In this spirit, the neutron time-of-flight spectrometer NEAT is designed to avoid spurious scattering within the flight path. From a neutron's point of view, the secondary spectrometer consists of the sample, the sample environment (SE), and the detectors; the entire setup is placed in a vacuum to minimize parasitic scattering from air. However, two main sources of spurious scattering exist: the sample environment and the detector tubes. The former varies as a broad range of sample conditions will be realized to cover a wide scope of applications, while the latter is indispensable as a container for the ${ }^{3} \mathrm{He}$ detection gas. 
If spurious scattering within the flight path is inevitable, it is convenient 45 to catch unwanted neutrons using shielding composed of elements with large 46 absorption cross sections, such as $\mathrm{Cd}, \mathrm{B}$ or Gd which are mounted between the 47 sample and the detectors. When the detectors surround the sample radially, as ${ }_{48}$ in the case of NEAT, thin shielding plates point to the centre of symmetry, i.e. 49 the position of the sample. With this arrangement, neutrons originating from 50 the sample are likely to pass while the chance of absorbing a neutron increases ${ }_{51}$ as its origin deviates from the sample position. Since the performance of the shielding relies on its geometry, a neutron becomes more likely to be absorbed 53 as a shielding approaches the scattering location. For this reason and to keep 54 matter within the flight path to a minimum, shields are preferably kept small and located as close as possible to the source of background.

In accordance with the two identified origins of parasitic scattering in NEAT's 57 setup, two kinds of shielding are considered. One set of shields composes the ${ }_{58}$ radial collimator which surrounds the sample and the sample environment to

catch scattering from the vicinity of the centre of symmetry. In strain scanning analysis, the device is used to define a small part of the sample [2-6], while in neutron diffractometers [7-14] and neutron time-of-flight spectrometers [15-21], its focus comprises the entire sample masking the sample environment. In the latter case, usually the device constantly rotates back and forth by a few degrees to average out the shadows cast from the vanes over the detectors [12]; if so, it is called an oscillating radial collimator (ORC). With regard to its construction, one usually relies on the focus of the radial collimator, i.e. the area that is visible from outside, but leaves the implementation of the focusing unsettled: neither an optimum length nor number of shields are specified. Moreover, a recent comparison [19] shows that radial collimators in operation lack a consistent correlation between the focused area and the beam size.

The second kind of shielding prevents the detection of neutrons that are back-scattered from other detectors, sometimes denoted as cross-talk. The detector shields are placed between the detector tubes and protrude towards the sample to some extent. Here, a variety of shielding concepts can be found, 
ranging from small shields between single detector tubes [17], to larger shields enclosing a certain number of tubes [22-25], to a combination of both [26]. This is remarkable since the general design of the instruments is similar in the sense that a large detector array surrounds a sample radially.

We conclude that although radial collimators and detector shielding are widely used, the theoretical framework lacks a general approach towards optimizing the performance by design. Here, we will introduce our proceedings to find the optimum shielding for the upgrade of the neutron time-of-flight spectrometer NEAT. To address this problem, the remainder of this paper is organized as follows. In Section 3, we will give the details of the methods employed, where we pursue a two-fold approach for oscillating radial collimators by investigating an analytical model in Section 3.1.1 supplemented by a Monte Carlo ray-tracing method in Section 3.1.2, while Section 3.2 is devoted to a stochastic treatment of detector shielding. Section 4 presents the results and discussions individually according to the ORCs in Section 4.1 and detector shielding setups in Section 4.2, before we close with a summary in Section 5.

\section{Models and methods}

To investigate the performance of the shielding, the visibility of certain areas within the instrument are calculated from a detector's point of view. For convenience, the shielding is assumed to absorb neutrons ideally, which means that the absorption process discards neutrons but neither causes scattering nor emits side products, such as gamma radiation. Since radial collimators and detector shielding aim at different regions of the instrument, they can be discussed independently from one another for the most part.

\subsection{Oscillating radial collimator}

NEAT is a promising tool for investigating samples of various states of matter, ranging from unordered fluids, to glasses featuring some short-range order, to single crystals with a strict symmetry. To optimize the background suppression on a universal level, we abstract from particular features of the sample and assume an isotropic incoherent scatterer. 


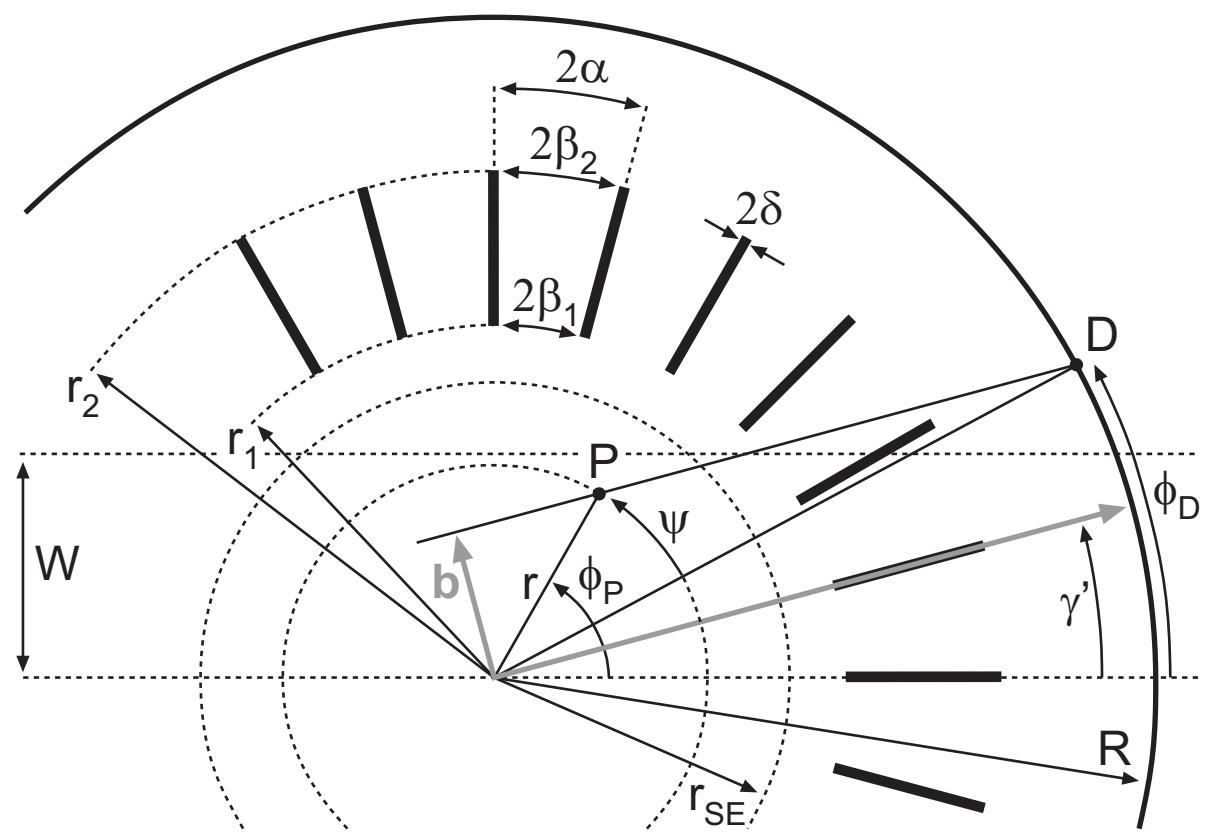

Figure 1: Sketch of the two-dimensional analytical model considering an infinitesimal thin annulus of radius $r$ inside an ORC defined by radii $r_{1}, r_{2}$ and repeat angle $2 \alpha$ surrounded by a radial detector system at the distance $R$ from the centre of symmetry. Rotating by $\gamma^{\prime}$, a trajectory becomes parallel to the abscissa at a distance $b$.

Surrounding the sample, an oscillating radial collimator is considered, which is defined by three parameters: the inner radius $r_{1}$, the outer radius $r_{2}$, and the repeat angle $2 \alpha$ that determines the angular spacing between adjacent collimator vanes. In fact, the thickness of the collimator vanes is also a crucial parameter, as it affects the transmission [5] and the sample's virtual centre of gravity [4]. However, its effect on the performance of ORCs is straightforward, and it results that the vanes should be as thin as possible. Throughout this study, a constant vane thickness of $2 \delta=0.16 \mathrm{~mm}$ is assumed, realized e.g. in the ENGIN diffractometer [5].

In the following, radial collimators are treated irrespective of detector shielding, as in case of the NEAT, the latter would be sufficiently distant from the area of interest and, thus, can be neglected regarding SE suppression. 


\subsubsection{Analytical model}

The effectiveness of a radial collimator is based on its ability to discriminate between trajectories in such a way the discrimination is independent of the vector component along the symmetry axis of the ORC (i.e. along its height). Thus, the two-dimensional geometric model proposed by Copley et al. [27] should faithfully reflect the fundamental physics. In the following, we briefly recapitulate the mathematical treatment of the so-called 'constant blade thickness ORC' and the resulting equations. However, the interested reader is referred to [27] whose notation is adopted.

Copley et al. consider an infinitesimal thin annulus of radius $r$ centred inside an ORC and surrounded by a radial detector, as shown in Fig. 1. If scattered at the annulus, a neutron must pass the collimator without hitting a collimator vane to reach the detector. With respect to the radial symmetry of the setup, we introduce polar coordinates to define the starting and end points of a neutron's trajectory. The location of the scattering event $P$ at the annulus is determined by the distance $r$ and angle $\phi_{P}$, whereas the point of detection $D$ is given by the detector radius $R$ and the detection angle $\phi_{D}$. A rotation by an angle of $\gamma^{\prime}$ reduces a given configuration of $P$ and $D$ to a trajectory which is parallel to the abscissa at a distance of $b$. As a result of this transformation, the distance $b$ determines the incident angle of the trajectory related to the collimator vanes and its probability of passing. Since NEAT's detector radius $R$ is large compared to the radius of the annulus $r$, the relevant angles are small and we may apply the small angle approximation to yield

$$
b=r \sin \psi
$$

where we switched to the detector's point of view since $\psi=\phi_{P}-\phi_{D}$ can be considered as the apparent angle in relation to $D$. The transmission $t$ of a 
trajectory can be written as a triangular function of the form

$$
\begin{aligned}
t(b) & =\beta_{1} / \alpha, & & |b| \leq \delta, \\
& =t_{0}\left(1-|b| / b_{0}\right), & & \delta \leq|b| \leq b_{0}, \\
& =0, & & |b| \geq b_{0},
\end{aligned}
$$

145 pass the ORC, written as

$$
b_{0}=\left(\beta_{1}+\beta_{2}\right) r_{c}
$$

146 with the characteristic radius $r_{c}$ given by

$$
r_{c}^{-1}=r_{1}^{-1}-r_{2}^{-1} .
$$

147

The parameter $\beta_{2}$ arising in Eqs. (3) and (4) defines one-half the angular spacing between adjacent collimator vanes at the outer radius $r_{2}$, whereas $\beta_{1}$ represents the corresponding quantity at the inner radius $r_{1}$.

To determine the amount of the annulus that is seen by a given detection point $D$, one can define a visibility function $V(r)$ as the transmission $t(b)$ averaged over all apparent angles $\psi$ :

$$
V(r)=\frac{1}{2 \pi} \int_{0}^{2 \pi} t(b) \mathrm{d} \psi .
$$

To evaluate $V(r)$, one has to consider a width of the neutron beam of $2 W$ that may illuminate the entire annulus or just a part of it. As long as $r \leq W$,

the entire annulus is illuminated by neutrons and the visibility function $V(r)$

is independent of the detection angle $\phi_{D}$. In this case, Eq. (6) becomes

$$
V(r)=\frac{2 t_{0}}{\pi}\left[\psi_{b}-\frac{\psi_{\delta} \delta}{b_{0}}+\frac{r\left(\cos \psi_{b}-\cos \psi_{\delta}\right)}{b_{0}}\right]
$$


where $\psi_{b}$ and $\psi_{\delta}$ depend either on $b_{0}$ written as

$$
\begin{aligned}
\psi_{b} & =\sin ^{-1}\left(\frac{b_{0}}{r}\right), & b_{0} & \leq r, \\
& =\frac{\pi}{2}, & b_{0} & \geq r,
\end{aligned}
$$

or on $\delta$ given through

$$
\begin{array}{rlrl}
\psi_{\delta} & =\sin ^{-1}\left(\frac{\delta}{r}\right), & & \delta \leq r, \\
& =\frac{\pi}{2}, & \delta \geq r .
\end{array}
$$

${ }_{157}$ If $r>W$, the treatment becomes more cumbersome, as arc sections may be ${ }_{158}$ visible (see Fig. 6 of [27]) and, thus, the visibility function depends on the 159 angle. As a result, only a certain range of $\psi$ is considered in computing the ${ }_{160}$ visibility function of Eq. (6), which may be written as

$$
V_{W}\left(r, \phi_{D}\right)=\frac{1}{\pi} \sum_{l=1}^{2} H\left(\psi_{l}^{+}-\psi_{l}^{-}\right) \int_{\psi_{l}^{-}}^{\psi_{l}^{+}} t(b) d \psi
$$

where $H\left(\psi_{l}^{+}-\psi_{l}^{-}\right)$is the Heaviside function

$$
\begin{array}{ll}
H\left(\psi_{l}^{+}-\psi_{l}^{-}\right)=0, & \psi_{l}^{+}-\psi_{l}^{-}<0 \\
H\left(\psi_{l}^{+}-\psi_{l}^{-}\right)=1, & \psi_{l}^{+}-\psi_{l}^{-} \geq 0
\end{array}
$$

determining whether an arc section is seen by the detection point $D$ or not. The boundaries of $\psi$ are given by

$$
\begin{aligned}
& \psi_{1}^{-}=\max \left(-\phi_{D}-\psi_{W},-\psi_{b}\right) \\
& \psi_{1}^{+}=\min \left(-\phi_{D}+\psi_{W}, \psi_{b}\right) \\
& \psi_{2}^{-}=\max \left(\pi-\phi_{D}-\psi_{W},-\psi_{b}\right) \\
& \psi_{2}^{+}=\min \left(\pi-\phi_{D}+\psi_{W}, \psi_{b}\right)
\end{aligned}
$$

where the operator 'max' or 'min' yields the larger or smaller term of the bracketed expressions, respectively, and $\psi_{W}$ is given by

$$
\begin{array}{rlrl}
\psi_{W} & =\sin ^{-1}\left(\frac{W}{r}\right), & W & \leq r, \\
& =\frac{\pi}{2}, & W & W r .
\end{array}
$$


Ideally, in neutron experiments, the sample diameter matches the beam dimensions, so that the scope $0 \leq r \leq W$ is assumed to belong to the sample. This area emits the signal of interest and its visibility is given by $V(r)$ of Eq. (7). Thus, the transmission $t_{a n}$ of an ORC can be written as

$$
t_{a n}=W^{-1} \int_{0}^{W} \frac{V(r)}{V_{W}^{n c}(r)} \mathrm{d} r
$$

where the denominator refers to the visibility function for the setup without ORC given through

$$
V_{W}^{n c}(r)=\frac{\psi_{W}}{\pi / 2} .
$$

An annulus larger than the sample, i.e. $W<r \leq r_{S E}$, corresponds to the sample environment, i.e. the source of parasitic scattering, given by $V_{W}\left(r, \phi_{D}\right)$ of Eq. (10). With it we may define the quality factor $Q_{W}$ of an ORC as

$$
Q_{W}\left(\phi_{D}\right)=\frac{\int_{0}^{W} r V(r) \mathrm{d} r}{\int_{W}^{r_{S E}} r V_{W}\left(r, \phi_{D}\right) \mathrm{d} r}
$$

where the integrals need some comment: the visibility functions yield the transmission of an annulus of radius $r$. Since we integrate over annuli of varying radius, the factor $r$ arises in the integrand to 'weight' an annulus according to its scattering probability that goes with the radius. This holds for the numerator and denominator, which implies that the SE is considered to be a continuous region with the same scattering characteristics as the sample. Moreover, the integral of the denominator is performed up to the outer radius of the sample environment $r_{S E}$. This takes a feature of the NEAT instrument into account where the entire setup of sample, sample environment, collimator, and detector is placed in a vacuum. As a result, the range $r_{S E} \leq r \leq r_{1}$ is assumed to bear a negligible scattering probability. Consequently, Eq. (16) can be considered as the signal-to-noise ratio (SNR) of a setup in vacuum at the detection angle $\phi_{D}$. By relating $Q_{W}\left(\phi_{D}\right)$ to the same quantity of a corresponding setup without collimator given through

$$
Q_{W}^{n c}=\frac{W^{2} / 2}{\left(r_{S E}^{2} / \pi\right) \sin ^{-1}\left(W / r_{S E}\right)+(W / \pi) \sqrt{r_{S E}^{2}-W^{2}}-W^{2} / 2}
$$


outer radius / thickness of $\mathrm{Al}$ ring $[\mathrm{mm}]$

\begin{tabular}{lcccc} 
sample environment & no. 1 & no. 2 & no. 3 & no. 4 \\
\hline Orange Cryofurnace (OF) & $76.5 / 1.5$ & $63.8 / 1.2$ & $41.5 / 1.0$ & $33.8 / 1.2$ \\
Orange Standard (OS) & $77.0 / 1.5$ & $62.5 / 1.2$ & $56.5 / 0.9$ & $26.2 / 1.2$ \\
Orange Maxi (OM) & $122.0 / 1.5$ & $100.0 / 1.6$ & $76.0 / 0.5$ & $51.5 / 1.5$ \\
Vertical Magnet (VM-2) & $234.0 / 2.0$ & $158.5 / 7.0$ & $54.5 / 1.9$ & $31.5 / 1.5$ \\
\hline
\end{tabular}

Table 1: Outer radius and thickness of $\mathrm{Al}$ rings (from outside to inside) mimicking the sample environments in Vitess calculations.

184

one may define the figure of merit as

$$
G_{a n}\left(\phi_{D}\right)=\frac{Q_{W}\left(\phi_{D}\right)}{Q_{W}^{n c}}
$$

yielding the factor by which the SNR is increased by using an ORC.

In general, $G_{a n}\left(\phi_{D}\right)$ depends on the detection angle, but for a specific instrument setup of fixed $W$ and $r_{S E}$, the figure of merit as well as its dependence on $\phi_{D}$ both are governed by the maximum impact parameter $b_{0}$ (a detailed derivation is given in Appendix A). Consequently, for an isotropic scatterer it makes sense to introduce a mean figure of merit by integrating $G_{a n}\left(\phi_{D}\right)$ over the detection angels cast as

$$
\left\langle G_{a n}\right\rangle=\frac{1}{\phi_{2}-\phi_{1}} \int_{\phi_{1}}^{\phi_{2}} G_{a n}\left(\phi_{D}\right) \mathrm{d} \phi_{D}
$$

where $\phi_{1}$ and $\phi_{2}$ are the lower and upper boundaries of the detection angle, respectively. $\left\langle G_{a n}\right\rangle$ can be considered as an ORC's impact on the signal-tonoise ratio of an isotropic scatterer achieved by a detection system.

In retrospect, we make some minor changes to the proposed treatment given in [27] by introducing (i) the outer dimension of the sample environment $r_{S E}$ in Eqs. (16) and (17), (ii) an angle-averaged figure of merit through Eq. (19), and (iii) the detector geometry via Eq. (19).

Throughout the calculations, we use $\phi_{1}=-81^{\circ}, \phi_{2}=143^{\circ}, r_{S E}=122 \mathrm{~mm}$ (outermost radius no. 1 of $\mathrm{OM}$ in Table 1 ), $2 W=30 \mathrm{~mm}$ and $2 \delta=0.16 \mathrm{~mm}$. The integrals are numerically evaluated with $\Delta r=0.1 \mathrm{~mm}$ and $\Delta \phi_{D}=0.001^{\circ}$. 


\subsubsection{Monte Carlo ray-tracing method}

To complement the analytical approach, we employed the Monte Carlo raytracing method implemented in the software package Vitess [28-32] (version 3.1), which treats three-dimensional neutron trajectories explicitly by time, position, direction, and wavelength. Vitess has a modular structure, where independent modules mimic the consecutive components of the instrument and assign probabilities to trajectories on the basis of random choices.

The simulation of NEAT's primary spectrometer comprises the cold source, a ballistic neutron guide [33] more than $60 \mathrm{~m}$ long including curved and focusing sections, and seven choppers described in more detail elsewhere [34]. The chopper configuration and focusing sections realize a beam size approximately $3 \mathrm{~cm}$ wide and $6 \mathrm{~cm}$ high at the sample position, with a wavelength of $5.1 \AA$ and a maximum divergence of about $\pm 1.5^{\circ}$.

The secondary spectrometer starts with the walls of a sample environment defined in Table 1 where the OM is employed if not otherwise mentioned. The discrete walls of the SE mimic aluminum with a macroscopic inverse total scattering length of $\Sigma_{s, A l}=0.09 \mathrm{~cm}^{-1}$ [35]. Therein a cylindrical sample of $3 \mathrm{~cm}$ diameter and $6 \mathrm{~cm}$ height is enclosed, roughly matching the beam dimensions in accordance with the preceding analytical model. To make contact with the experimental conditions, the powder sample module is used with the 'incoherent scattering' and 'treat all neutrons' option, to ensure that the walls of the SE behind the sample are exposed to the incident beam. The sample is assumed to be a $10 \%$ incoherent scatterer with $\Sigma_{s, s}=0.045 \mathrm{~cm}^{-1}$, i.e. the chance for scattering a neutron incoherently is 0.1 while the coherent cross section is zero. However, the sample neither takes into account self-shielding nor multiple scattering, but a neutron may be scattered at the sample and at different components of SE. Leaving the sequence SE-sample-SE, neutrons pass or get absorbed by an oscillating radial collimator of variable design but constant vane thickness of $2 \delta=0.16 \mathrm{~mm}$. Finally, trajectories may reach the cylindrical detector system of $3 \mathrm{~m}$ radius made up of detector tubes of $2.54 \mathrm{~cm}$ diameter and $2 \mathrm{~m}$ 
height, which cover the horizontal range from $\phi_{1}=-81^{\circ}$ to $\phi_{2}=143^{\circ}$ related to the incident beam. Trajectories that are neither scattered at the sample nor the sample environment are absorbed by a beam stop located before the detectors.

To make contact with the analytical model, the transmission is defined as

$$
\left\langle t_{m c}\right\rangle=\frac{1}{\phi_{2}-\phi_{1}} \int_{\phi_{1}}^{\phi_{2}} t_{m c}\left(\phi_{D}\right) \mathrm{d} \phi_{D}
$$

where $t_{m c}=I_{s, c}\left(\phi_{D}\right) / I_{s, n c}\left(\phi_{D}\right)$ refers to the ratio of intensities originating from the sample in the setup with $\operatorname{ORC}\left(I_{s, c}\right)$ and without collimator $\left(I_{s, n c}\right)$. According to Eqs. (16)-(19) the average figure of merit is

$$
\left\langle G_{m c}\right\rangle=\frac{1}{\phi_{2}-\phi_{1}} \int_{\phi_{1}}^{\phi_{2}} G_{m c}\left(\phi_{D}\right) \mathrm{d} \phi_{D}
$$

with $G_{m c}\left(\phi_{D}\right)=S N R_{c}\left(\phi_{D}\right) / S N R_{n c}\left(\phi_{D}\right)$ being the ratio of signal-to-noise ratio of the setup with ORC to the SNR of the situation without ORC. The signal-to-noise ratio is computed through

$$
\operatorname{SNR}\left(\phi_{D}\right)=\frac{I_{s}\left(\phi_{D}\right)}{I_{S E}\left(\phi_{D}\right)}
$$

where $I_{s}\left(\phi_{D}\right)$ is the intensity originating from the sample while intensity $I_{S E}\left(\phi_{D}\right)$ comprises the trajectories that are scattered at the sample environment (at least once). All the integrals of Eqs. (20) and (21) were numerically evaluated using $\Delta \phi_{D}=5^{\circ}$.

\subsection{Detector shielding}

Although the constructions are related to one another, the treatment of detector shielding differs in principle from that of radial collimators because the trajectories of interest start and end within the same region. As a consequence, the quantification of the cross-talk requires the computation of the self-visibility

$V_{s}$ of the detection area, which is performed stochastically through

$$
V_{s}=\frac{I_{c}}{I_{t}}
$$

252 where $I_{t}$ is the total number of created trajectories determined by a random starting point at the detection area and a random flight direction while $I_{c}$ is the number of trajectories that hit the detection area. 


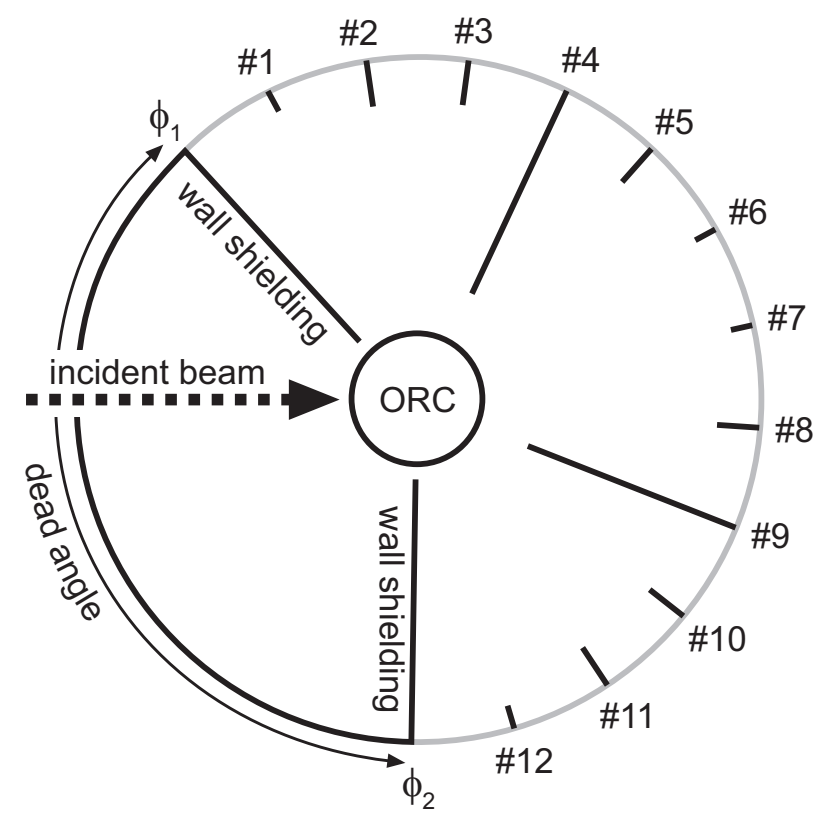

Figure 2: Sketch of NEAT's geometry (top view) used to compute the self-visibility of the detector system (grey). Black lines resemble the neutron absorbing instrument parts, comprising instrument walls, dead angle of $136^{\circ}$, oscillating radial collimator, and shielding. Here, 12 module shields of various lengths are shown.

Consider NEAT's detector system covering a part of a cylinder of 3 m radius, $2 \mathrm{~m}$ height and realizing a total detection angle of $\Delta \phi=\phi_{2}-\phi_{1}=224^{\circ}$, sketched in Fig. 2. The detection area is bounded by walls at $\phi_{2}, \phi_{1}$ that protrude 2.20 $\mathrm{m}$ toward the sample position and that enclose a dead angle of $136^{\circ}$. Starting at the detection area, the shielding may protrude towards the sample position to intercept the line-of-sight between parts of the detection system. The shields are rectangular and match the height of the detectors, while their thickness is assumed to be negligible.

Besides the shielding, a radial collimator can decrease $V_{s}$ since it is unlikely that a neutron will pass its arrangement of neutron absorbing vanes. If in place, a cylindrical region defined by the ORC's outer radius $r_{2}=578 \mathrm{~mm}$ around the sample position is blocked, where its height is assumed to match the height of 267 the detectors as NEAT's design prevents neutrons from passing above or below 
the radial collimator. Since the complexity of the instrument geometry is kept to an absolute minimum, neither how the detector system is made up out of the tubes, nor the details of the radial collimator other than its outer radius are considered.

Assuming that the detection area is homogeneously illuminated by a sample's isotropic scattering and that the detector tubes back-scatter isotropically as well, the self-visibility function $V_{s}$ of the three-dimensional detector system is computed through a Monte Carlo algorithm of the following form: (i) Generate a random starting position within the detection area for a neutron trajectory; (ii) Create a random flight direction for the trajectory; (iii) If a radial collimator is in place, check whether the trajectory intersects the ORC's outer radius $r_{2}$; (iv) Check whether the trajectory intersects a detector shield; (v) If the trajectory neither intersects the ORC nor the shielding, check whether the trajectory intersects the detection area (at a position different from the starting point); (vi) repeat steps (i) to (v).

The number of trajectories that intersect the detection area (a second time) without being blocked by another instrument part is proportional to the intensity of detected cross-talk, and denoted by $I_{c}$, while the remaining trajectories are assumed to be absorbed. The total number of created trajectories $I_{t}$ was on the order of $10^{6}$ to $10^{9}$ in this study.

\section{Results and discussion}

Aside from the sample, parts of the sample environment are directly exposed to the incident beam, producing a substantial portion of the parasitic scattering. Disregarding a sample's absorption, the amount of spurious scattering emitted from the $\mathrm{SE}$ would be constant and the signal-to-noise ratio depend solely on the scattering characteristics of the sample. For this reason, figures of merit instead of signal-to-noise ratios are employed to keep the discussion on a universal level.

\subsection{Suppression of sample environment scattering}

The analytical treatment of 3.1.1 reveals that an ORC is unable to make a clear distinction between the sample and the sample environment, i.e. the 


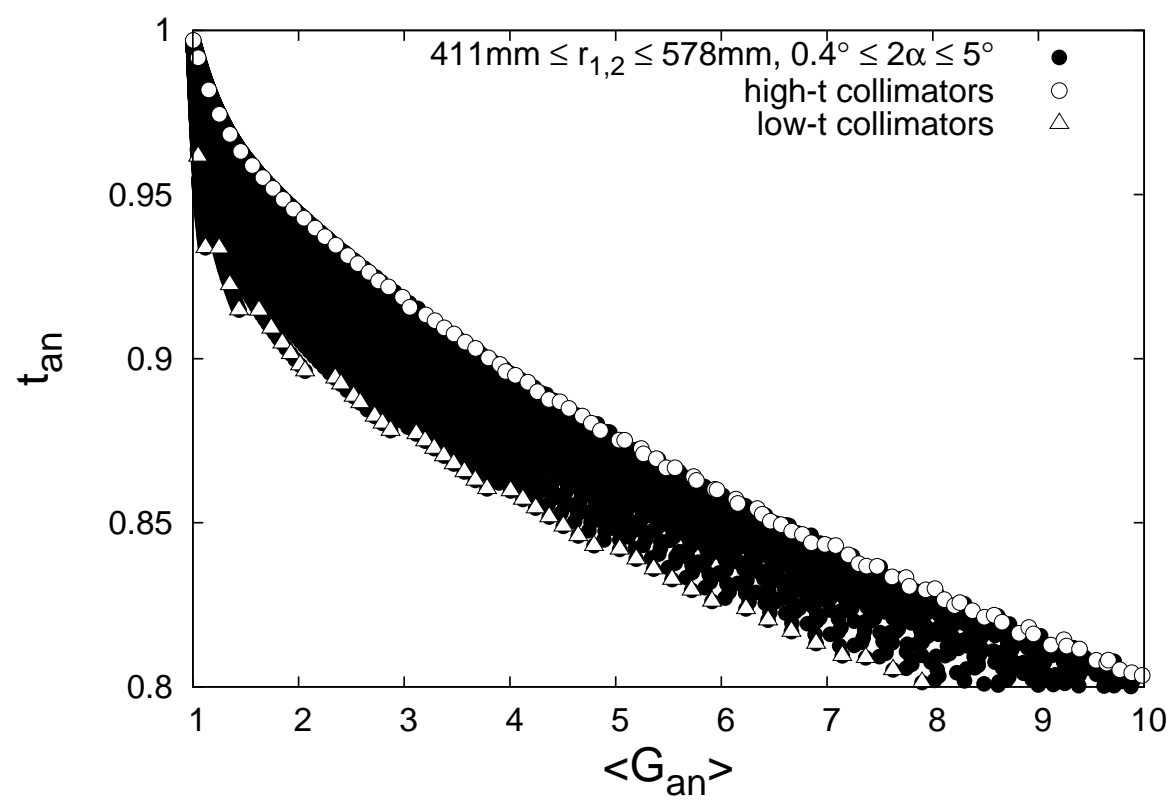

Figure 3: Transmission $t_{a n}$ as a function of figure of merit $\left\langle G_{a n}\right\rangle$ derived from the analytical model for collimators with $411 \mathrm{~mm} \leq r_{1}, r_{2} \leq 578 \mathrm{~mm}$ and $0.4^{\circ} \leq 2 \alpha \leq 5.0^{\circ}$. Unfilled dots indicate the high- $t$ collimators with the largest possible transmission, whereas unfilled triangles denote low- $t$ collimators with the smallest transmission possible for a given figure of merit. Interstitials within the $t_{a n}-\left\langle G_{a n}\right\rangle$ space accounts for the intervals $\Delta r_{1,2}=5 \mathrm{~mm}$ and $\Delta 2 \alpha=$ $0.1^{\circ}$ used throughout the calculations.

visibility function has a non-vanishing contribution for $r>W$. This becomes apparent by comparing Eqs. (A.1)-(A.3) since $V(r)$ and $V_{W}\left(r, \phi_{D}\right)$ are both functions of $b_{0}$; thus, the focus of an ORC contributes to the visibility of the SE as well. As a consequence, background suppression is accompanied by a loss in signal. To address the effectiveness of an ORC, the transmission and figure of merit are crucial quantities, as they quantify the trajectories originating from the sample and their relation to the parasitic scattering.

The formalism of the analytical model is computationally undemanding and a large number of ORCs with the spatial characteristics of the NEAT spectrometer can be simulated. Here, the inner radius $r_{1}$ must be larger than the sample environment used, while the sample chamber limits the outer radius $r_{2}$. To meet the spatial requirements of the instrument, the radii were restricted to 


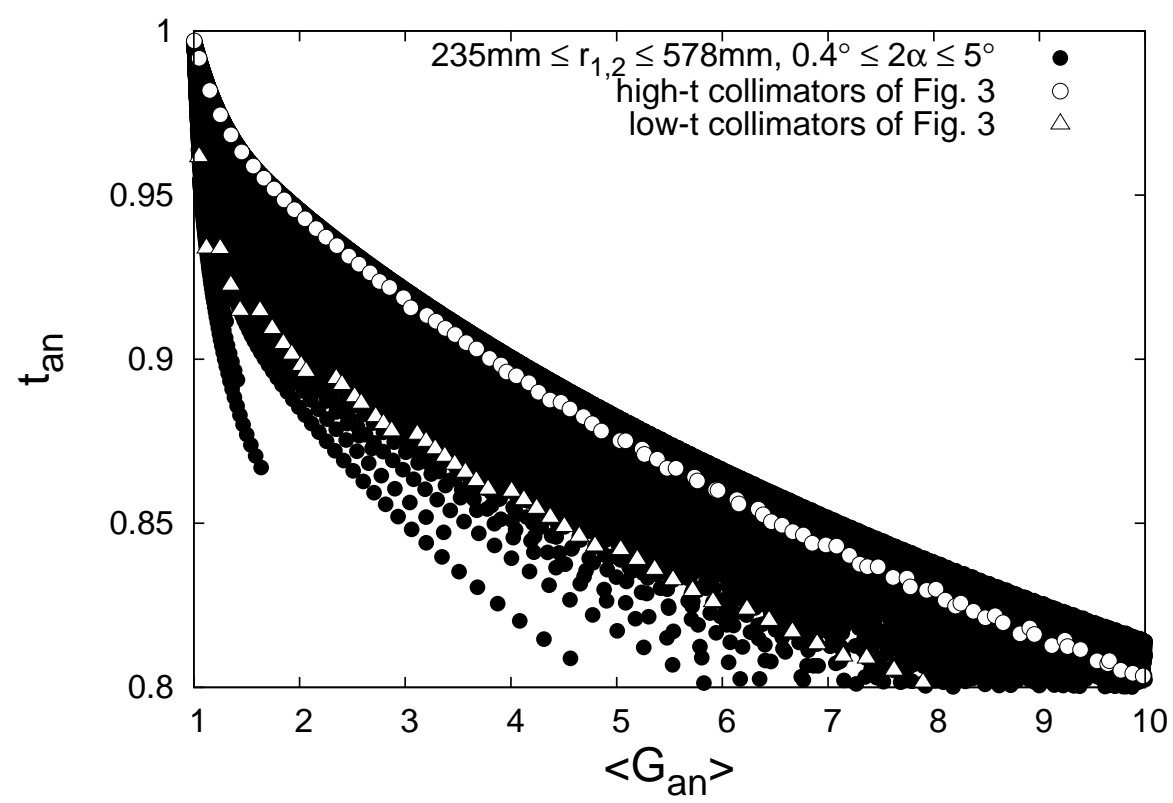

Figure 4: As Fig. 3 but the parameter space is increased to $235 \mathrm{~mm} \leq r_{1}, r_{2} \leq 578 \mathrm{~mm}$ and $0.4^{\circ} \leq 2 \alpha \leq 5.0^{\circ}$. Unfilled dots and triangles indicate the high- $t$ and low- $t$ collimators of Fig. 3 , respectively. 
the range $411 \mathrm{~mm} \leq r_{1,2} \leq 578 \mathrm{~mm}$. The minimum repeat angle $2 \alpha$ is restricted by technical feasibility and is assumed to be $0.4^{\circ}$, corresponding roughly to the angular spacing realized in the D20 instrument [36]. The maximum limit of $5^{\circ}$ is arbitrarily chosen, based on the fact that the collimation effect vanishes with increasing repeat angle. Intervals of $\Delta 2 \alpha=0.1^{\circ}$ and $\Delta r_{1,2}=5 \mathrm{~mm}$ with $r_{1}<r_{2}$ are employed to cover the parameter space homogeneously, thus modeling more than $2 \times 10^{4}$ collimators.

Figure 3 shows the transmission as a function of the figure of merit for collimators with $t_{a n} \gtrsim 0.8$. Here, a single point represents an ORC with a certain set of parameters $r_{1}, r_{2}$ and $2 \alpha$. The rather broad distribution reveals that a given figure of merit can be achieved at different transmissions. This allows one to distinguish between 'high-t' and 'low- $t$ ' collimators, where the former realize the largest possible transmission, while the latter realize the lowest transmission at the same figure of merit. In this spirit, Fig. 3 indicates the high- $t$ collimators by unfilled dots, and low- $t$ collimators by unfilled triangles. The discrepancy in transmission between high- $t$ and low- $t$ collimators is up to a few percent and, thus, rather small. However, the spread depends on the parameter space considered. For example, by decreasing the lower boundary of the radius from $411 \mathrm{~mm}$ to $235 \mathrm{~mm}$ (while keeping $r_{S E}=122 \mathrm{~mm}$ ), the discrepancies increase up to $10 \%$, as shown in Fig. 4. A comparison of Fig. 4 with Fig. 3 reveals that mainly the lower boundary of the covered $t_{a n}-\left\langle G_{a n}\right\rangle-$ space is shifted to lower transmissions, while the upper boundary increases only slightly to larger transmissions. In other words, by allowing smaller radii, mostly inferior ORCs become accessible, revealing that an additional ORC optimized for smaller sample environments such as OM, OF or OS (see Table 1) would be of limited advantage.

The reason for this becomes apparent when we extract recipes for designing collimators with the largest and smallest possible transmissions from Fig. 3. In Fig. 5, the parameters $r_{1}, r_{2}$ and $2 \alpha$ of the high- $t$ and low- $t$ collimators are plotted as functions of the figure of merit. The design of both collimator types follows a general principle where two parameters are fixed and the third is used 


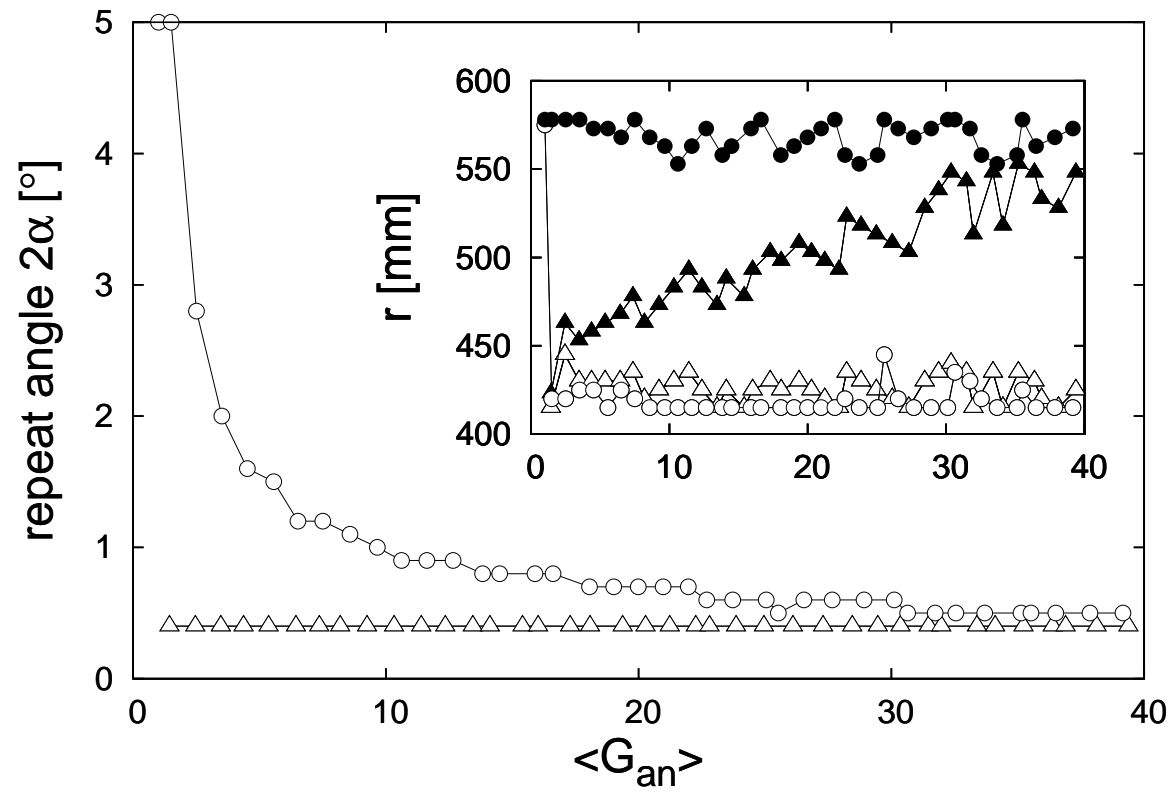

Figure 5: Repeat angle $2 \alpha$ is plotted in dependence on $\left\langle G_{a n}\right\rangle$ for the high- $t$ collimators (dots) and low- $t$ collimators (triangles) of Fig. 3. Inset: Inner radius $r_{1}$ (unfilled symbols) and outer radius $r_{2}$ (filled symbols) are shown as functions of $\left\langle G_{a n}\right\rangle$ for the high- $t$ (dots) and low- $t$ (triangles) collimators of Fig. 3. Outliers and variations mainly stem from limitation and quantization of parameter space. 


\begin{tabular}{ccccccccc}
\hline & & & & \multicolumn{3}{c}{ figure of merit } & \multicolumn{2}{c}{ transmission } \\
no. & $r_{1}[\mathrm{~mm}]$ & $r_{2}[\mathrm{~mm}]$ & $2 \alpha\left[^{\circ}\right]$ & $2 b_{0}[\mathrm{~mm}]$ & $\left\langle G_{a n}\right\rangle$ & $\left\langle G_{m c}\right\rangle$ & $t_{a n}$ & $\left\langle t_{m c}\right\rangle$ \\
\hline 1 & 440 & 473 & 0.4 & 83.6 & 5.04 & 9.37 & 0.842 & 0.810 \\
2 & 445 & 488 & 0.5 & 84.7 & 4.96 & 9.26 & 0.853 & 0.819 \\
3 & 460 & 518 & 0.6 & 83.3 & 5.06 & 9.47 & 0.858 & 0.822 \\
4 & 425 & 483 & 0.7 & 84.0 & 5.01 & 9.41 & 0.861 & 0.828 \\
5 & 450 & 528 & 0.8 & 83.1 & 5.09 & 9.66 & 0.865 & 0.831 \\
6 & 415 & 498 & 1.0 & 85.2 & 4.92 & 9.10 & 0.871 & 0.838 \\
7 & 430 & 573 & 1.4 & 83.1 & 5.08 & 9.61 & 0.874 & 0.842 \\
8 & 420 & 578 & 1.6 & 84.8 & 4.94 & 9.21 & 0.878 & 0.845 \\
\hline
\end{tabular}

Table 2: Results of the analytical model and Vitess calculations for arbitrarily chosen collimators from Fig. 3 with $\left\langle G_{a n}\right\rangle=5.0 \pm 0.1$.

to adjust the figure of merit: high- $t$ ORCs share the longest blades possible and use the repeat angle to define the figure of merit, while low- $t$ collimators tune $\left\langle G_{a n}\right\rangle$ by increasing the outer radius and keep the repeat angle and inner radius as small as possible. However, a decrease in the inner radius increases the shadow effect of the collimator vanes [37]. This affects low- $t$ collimators more than high- $t$ collimators because low- $t$ collimators have a larger number of collimator vanes due to the minimum repeat angle. As a result, the $t_{a n}-\left\langle G_{a n}\right\rangle-$ space mainly increases by decreasing its lower boundary.

Table 2 lists arbitrarily chosen collimators from Fig. 3 which realize comparable figures of merit of $\left\langle G_{a n}\right\rangle=5.0 \pm 0.1$ by varying their design. The collimators share a similar maximum impact parameter $b_{0}$ given in Eq. (4) and, thus, focus on a similar area of diameter $2 b_{0}$. The collimator designs were used as the input for Vitess simulations, and the resulting transmissions $\left\langle t_{m c}\right\rangle$ and figures of merit $\left\langle G_{m c}\right\rangle$ complete the table. Regarding the sequence from no. 1 to 8 , $\left\langle t_{m c}\right\rangle$ increases monotonically in good agreement with the $t_{a n}$ of the analytical model, although the transmissions of the Vitess calculations are smaller

57 than their counterparts derived from the analytical model. The average figure ${ }^{58}$ of merit $\overline{\left\langle G_{m c}\right\rangle}=9.4 \pm 0.3$ taken from the Monte Carlo method is significantly larger than $\left\langle G_{a n}\right\rangle$ as the models differ in the definition of the SE; the former considers an arrangement of discrete aluminum rings, while the latter assumes 


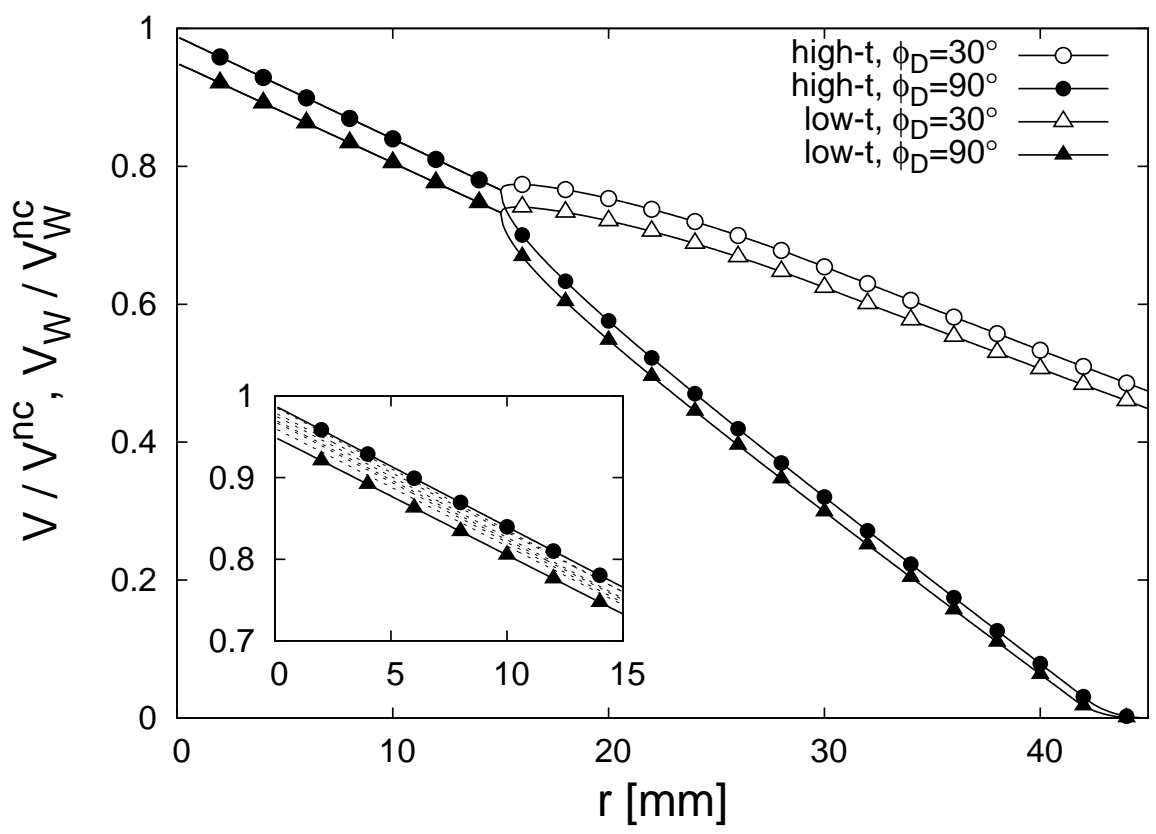

Figure 6: $V / V^{n c}$ for $r \leq W$ and $V_{W} / V_{W}^{n c}$ for $r>W$ (with $W=15 \mathrm{~mm}$ ) as functions of radius $r$. Dots correspond to high- $t$ collimator no. 8 while triangles denote the low- $t$ collimator no. 1 of Table 2. Regarding the angular dependence of $V_{W}$, filled symbols indicate a detection angle of $\phi_{D}=90^{\circ}$ whereas unfilled symbols indicate $\phi_{D}=30^{\circ}$. Inset: In addition, collimators no. 2 to 7 (from top to bottom) of Table 2 are plotted as dashed lines for the range $0 \mathrm{~mm} \leq r \leq 15 \mathrm{~mm}$.

a continuous region around the sample as the SE. The different treatment of the SE results in different absolute values of the figure of merit, but comparable spreads of $\pm 3 \%$ and $\pm 2 \%$, respectively, reveal that the selected ORCs share the same figure of merit regardless of the method applied.

No. 1 and no. 8 of Table 2 correspond to the low- $t$ collimator and the high- $t$ collimator of $\left\langle G_{a n}\right\rangle=5.0 \pm 0.1$, respectively, whose visibility functions are plotted in Fig. 6. $V(r)$ as well as $V_{W}(r)$ of the high- $t$ collimator are larger than those of the low- $t$ collimator, realizing a larger transmission at the same figure of merit, while the visibility functions of collimators no. 2-7 lie in between, shown as dashed lines in the inset. The transmission and figure of merit for the high- $t$ as well as the low- $t$ collimator are calculated using Vitess and are plotted as 32 functions of the detection angle in Fig. 7. Both radial collimators share the angular dependence of the figure of merit while the transmission of the high- $t$ 


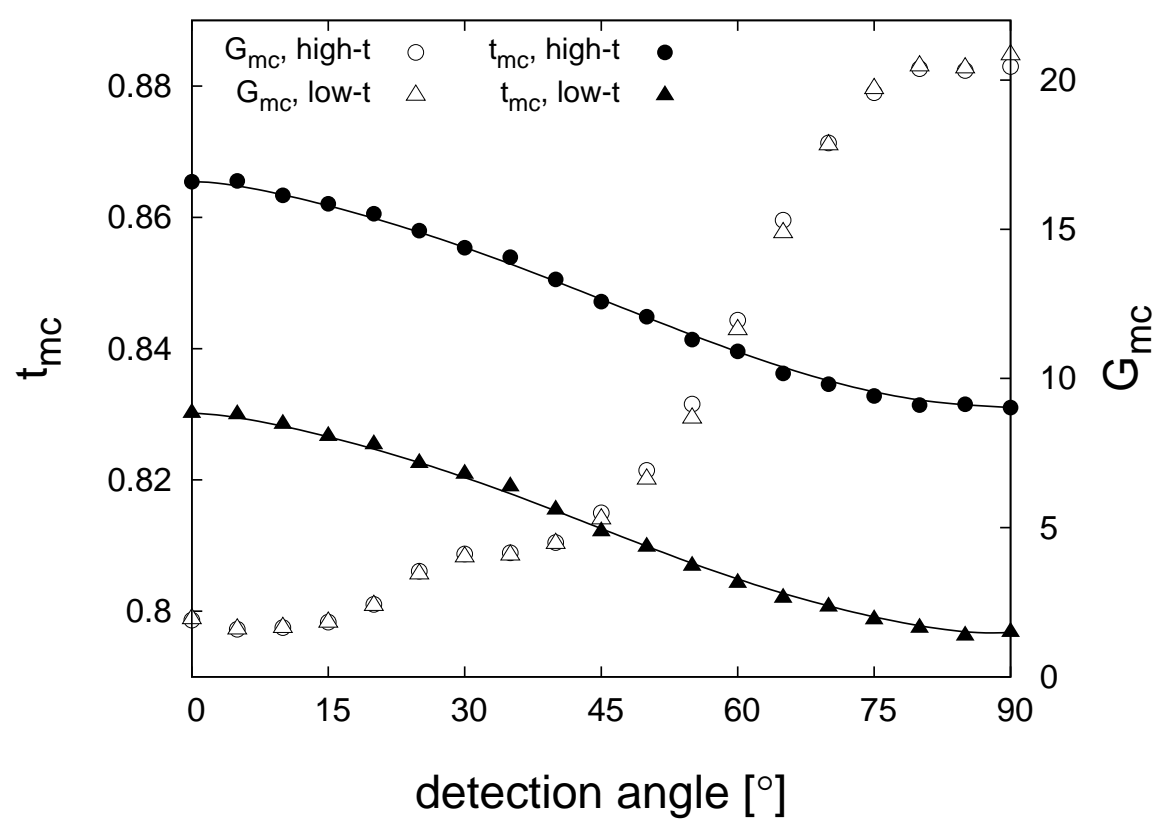

Figure 7: Angle-resolved transmission $t_{m c}$ (filled symbols) and figure of merit $G_{m c}$ (unfilled symbols) as functions of the detection angle for the low- $t$ (triangles) and the high- $t$ collimators (dots) sharing $\left\langle G_{a n}\right\rangle=5.0 \pm 0.1$ (no. 1 and 8 of Table 2). 
collimator is in general larger than the transmission of the low- $t$ collimator, i.e. the former is shifted by a constant from that of the latter, in agreement with Fig. 6. The angular dependence of the transmission, in contrast to $V(r)$ of Eq. (14), arises from the fact that in the Vitess calculations, the effective beam is neither ideally homogeneous nor exactly defined, as assumed by the analytical model. In this case, minor deviations from the ideal cause significant angle dependencies (see Fig. 8a of [27]). This is a consequence of the convolution of the triangular transmission function of Eq. (2) with the intensity distribution of the beam at different detection angles, where both functions are maximum at the centre. At smaller detection angles, the maxima of both functions overlap with one another to a greater extent and result in larger effective transmissions than at angles around $90^{\circ}$. As a result, the analytical model overestimates the transmissions $t_{a n}$ compared to the $\left\langle t_{m c}\right\rangle$ of the Vitess calculations in general, as Table 2 suggests.

Fig. 8 shows the transmission as a function of the figure of merit for the low- $t$ and high- $t$ collimators derived from Fig. 3. In addition, the corresponding Vitess calculations are included, which confirm the discrepancy in transmission between the high- $t$ and low- $t$ collimators predicted by the analytical model although $\left\langle G_{m c}\right\rangle$ depends significantly on the SE used. The reason for this dependence becomes apparent from Fig. 9, which plots $\left\langle t_{m c}\right\rangle$ against $\left\langle G_{m c}\right\rangle$ for the high- $t$ collimators for various SEs. Since the Vitess calculations employ more realistic sample environments, composed of discrete sections of matter, the figure of merit varies considerably with the details of the SE: $\left\langle G_{m c}\right\rangle$ is sensitive to the spatial distribution of matter, as the visibility function of Eq. (10) decays with $r$ (see Fig. 6). The two innermost walls of the OM sample environment are more distant to the sample position than the walls of the other SEs do (compare rings no. 3 and 4 in Table 1), resulting in rather large figures of merit. However, the transmission of a collimator stems from the sample and, thus, is independent of the SE, as emphasized by the dashed line in Fig. 9 indicating the average transmission of collimator no. 8 of Table 2. As a result, it is convenient to distinguish between the various high- $t$ collimators by transmission rather than by 


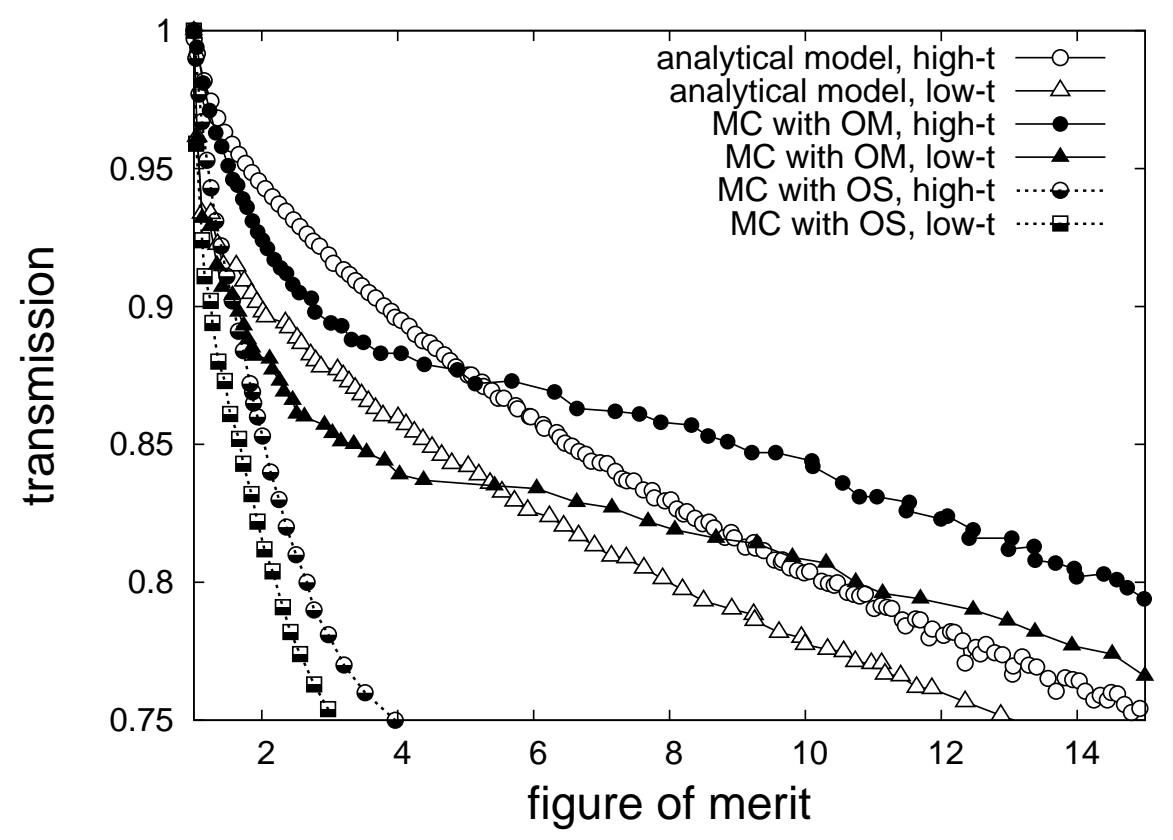

Figure 8: $t_{a n}$ vs. $\left\langle G_{a n}\right\rangle$ for the analytical model and $\left\langle t_{m c}\right\rangle$ vs. $\left\langle G_{m c}\right\rangle$ in case of Vitess calculations are plotted. Filled dots and filled triangles correspond to Vitess simulations of high- $t$ and low- $t$ collimators, respectively, in conjunction with the OM sample environment, while half-filled dots and half-filled squares represent Vitess results for high- $t$ and low- $t$ collimators, respectively, combined with the OS sample environment. Irrespective of the different courses, the deviations resemble the discrepancies between the high- $t$ (unfilled dots) and low- $t$ collimators (unfilled triangles) of the analytical model (same data as Fig. 3). 


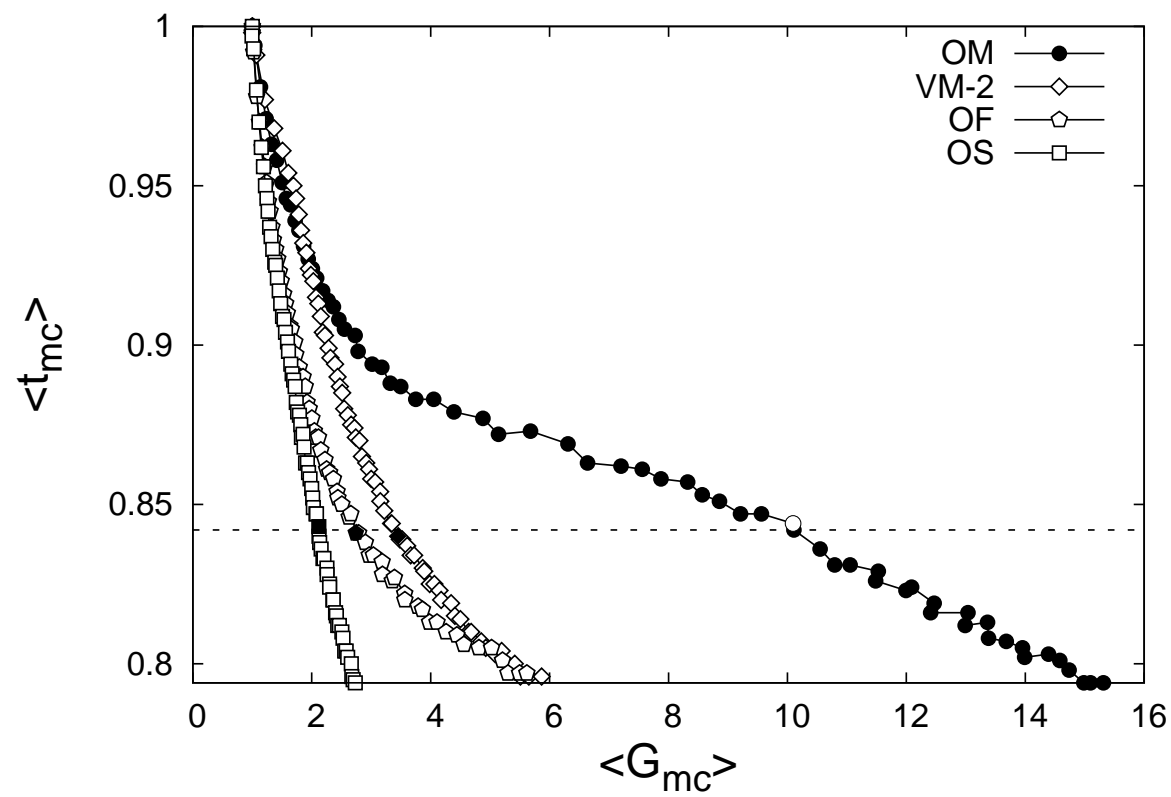

Figure 9: Transmission vs. figure of merit for high- $t$ collimators with different sample environments defined by Table 1 . Filled inverted symbols indicate collimator no. 8 of Table 2 for various SEs. The dashed line indicates its average transmission. The OM, OF, and OS sample environments have similar amounts of aluminum in the beam (see Table 1) and, thus, have similar signal-to-noise ratios at $\left\langle G_{m c}\right\rangle=1$, but their background suppression varies by up to a factor of 5 for collimator no. 8. Data for the OM and OS sample environment are taken from Fig. 8.

figure of merit.

The signal-to-noise ratio, and therefore the figure of merit, are crucial quantities as long as the signal significantly exceeds the spurious SE scattering. Here, the maximum figure of merit is limited by the basic background level that originates from imperfections of the instrument (e.g. dark counts), and which prevents the ORC from increasing the SNR beyond a (usually unknown) value. However, when the signal becomes comparable to the background, the loss in transmission accompanying a gain in the figure of merit has the opposite effect: it impedes the detection [38].

As in the case of the simulations where the sample size is fixed, one may think of the sample as a small amount of the specimen of interest that is homogeneously distributed in a matrix of constant volume and negligible scattering 


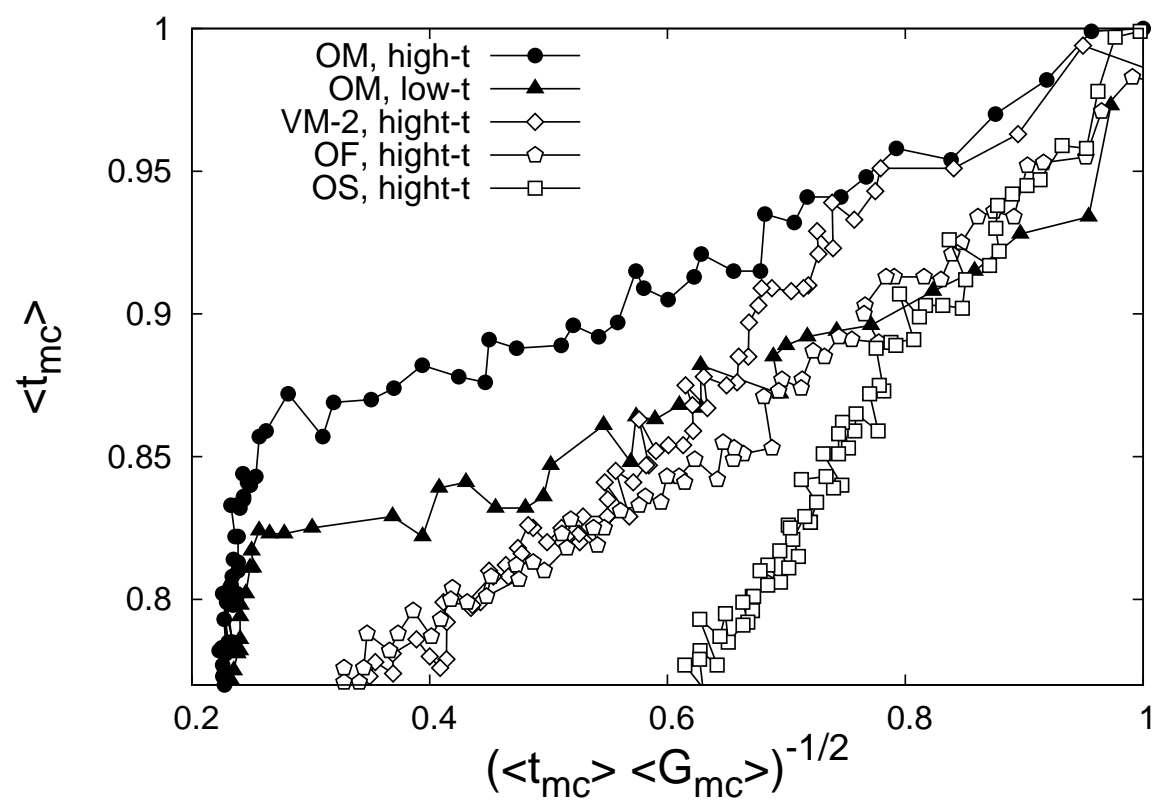

Figure 10: Same data as Fig. 9, but here the transmission is plotted as a function of $\left(\left\langle t_{m c}\right\rangle\left\langle G_{m c}\right\rangle\right)^{-1 / 2}$, which is proportional to the detection limit as in Eq. (24). Additionally, data for low- $t$ collimators under the OM sample environment are shown (taken from Fig. 8). 
characteristics, such as a dilute solution. The smallest concentration of the specimen whose signal can be distinguished from the background is the socalled detection limit $\left\langle C_{D L}\right\rangle$, which is independent of the considered dimension; for instance, it may concern a Bragg peak along the scattering vector $\mathrm{Q}$ or a Gauss-like distribution on the time-of-flight axis.

Assuming the background varies marginally in the vicinity of a peak, the detection limit $\left\langle C_{D L}\right\rangle$ of the signal measured for a certain time period can be written as [39]

$$
\begin{aligned}
\left\langle C_{D L}\right\rangle & =A\left(\frac{\left\langle I_{s}\right\rangle^{2}}{\left\langle I_{S E}\right\rangle}\right)^{-\frac{1}{2}} \\
& \approx A\left(\left\langle t_{m c}\right\rangle\left\langle G_{m c}\right\rangle\right)^{-\frac{1}{2}}
\end{aligned}
$$

where $A$ is constant for the system under consideration (incorporating the properties of the sample and the details of the detection process), and $\left\langle I_{s, S E}\right\rangle$ denotes intensities of sample or sample environment, integrated over the detection angle $\phi_{D}$ cast as

$$
\left\langle I_{s, S E}\right\rangle=\int_{\phi_{1}}^{\phi_{2}} I_{s, S E}\left(\phi_{D}\right) \mathrm{d} \phi_{D}
$$

Since $\left\langle I_{s}\right\rangle$ and $\left\langle I_{S E}\right\rangle$ both involve an integration over the detection angle $\phi_{D}$ which would prevent $\left\langle C_{D L}\right\rangle$ from being expressed in terms of $\left\langle t_{m c}\right\rangle$ and $\left\langle G_{m c}\right\rangle$, the proportionality of Eq. (24) is not strictly valid for the Monte Carlo method. However, throughout the simulations the dependence on $\phi_{D}$ is small for $\left\langle t_{m c}\right\rangle$ compared to $\left\langle G_{m c}\right\rangle$ as shown in Fig. 7 and, thus, the approximation of Eq. (24) is reasonable.

Fig. 10 shows the transmission vs. $\left(\left\langle t_{m c}\right\rangle\left\langle G_{m c}\right\rangle\right)^{-1 / 2}$ and thus, as in Eq. (24), as a function of the detection limit. Concerning the high- $t$ collimators under the OF, OS, and VM-2 sample environments, a decrease in transmission decreases the detection limit continually in the considered range. Under the OM sample environment, a reduction of transmission is accompanied by a drop in the detection limit down to a transmission of about 0.85. From there on, a decrease of transmission (and increase of the figure of merit) fails to improve the detection limit significantly. Concerning the detection limit at the same transmission, Fig. 
10 reveals that high- $t$ collimators (filled circles) can grant significant advances:

444 up to $50 \%$ smaller concentrations can be detected compared to low- $t$ collima-

445 tors (filled triangles). However, below a transmission of about 0.82 , the low- $t$

446 collimators almost attain the detection limit of their high- $t$ counterparts.

${ }_{447}$ Here, again one may meet the basic background level of the instrument,

448 which prevents the ORC from decreasing the detection limit beyond a certain

449 value; in fact, further improvement of the ORC's figure of merit beyond this

${ }_{450}$ value would increase $\left\langle C_{D L}\right\rangle$ as the background remains constant while the trans-

451 mission is decreased. However, NEAT's basic background level is expected to lie

${ }_{452}$ below the considered range due to the unperturbed flight path of the secondary

453 spectrometer.

As a result, collimator no. 8 of Table 2 with $\left\langle t_{m c}\right\rangle \approx 0.85$ and $\left\langle G_{m c}\right\rangle \approx 10$ under the OM, as the standard sample environment, can be considered to be close to optimal regarding the transmission and the detection limit. Following the recipe for designing high- $t$ collimators derived from Fig. 5 , we refine the design to compensate for the quantization of the parameter space. Realizing the longest blades possible, the radii are modified to $r_{1}=411 \mathrm{~mm}$ and $r_{2}=578 \mathrm{~mm}$ while the repeat angle is set to $2 \alpha=1.63^{\circ}$. The latter is that multiple of the angular detector spacing which will allow a static operation of the radial collimator where its orientation is fixed so that shadows cast from the collimator vanes match the gaps between detectors, which can grant another $2 \%$ in transmission.

The vanes of the radial collimator are intended to consist of $0.1 \mathrm{~mm}$ thick Kapton foils covered on both sides by a thin layer of $\mathrm{Gd}_{2} \mathrm{O}_{3}$. As suggested in [19], the vanes that are exposed to the incident beam will be omitted since they

We now focus on neutrons that leave the radial collimator and head for the ${ }_{471}$ detectors. Before triggering the ${ }^{3} \mathrm{He}$ detection process, a neutron may scatter ${ }_{472}$ at the tube's wall. Since detectors are not exposed to the incident beam, the 


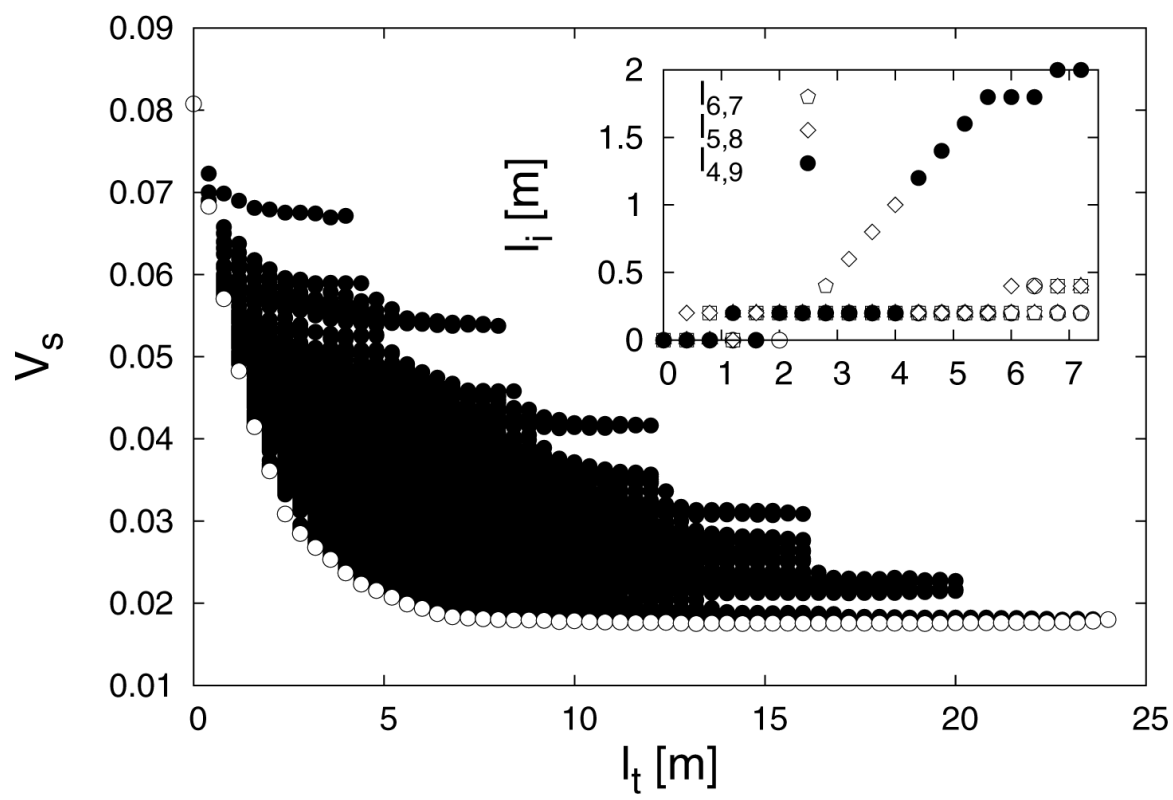

Figure 11: Self-visibility $V_{s}$ as a function of total shielding length $l_{t}$ for 12 module shields in the range $0 \mathrm{~cm} \leq l_{i} \leq 200 \mathrm{~cm}$ with $\Delta l_{i}=20 \mathrm{~cm}$ in conjunction with ORC. Unfilled dots mark low- $V_{s}$ module shields, which suppress cross-talk efficiently, i.e. shielding with the smallest $V_{s}$ at a given $l_{t}$. Inset: Length of individual module shields $l_{i}$ vs. $l_{t}$ for low- $V_{s}$ configurations of the main plot in the range $0 \mathrm{~m} \leq l_{t} \leq 7.5 \mathrm{~m}$. Module shields are numbered according to Fig. 2 .

resulting spurious scattering is expected to be one or two orders of magnitude 
ous scattering (particular within the neutron's flight path), the total shielding length $l_{t}$ of a configuration of $N_{s}$ shields is of interest, and can be written as

$$
l_{t}=\sum_{i}^{N_{s}} l_{i}
$$

${ }_{487}$ provided that the height of the shields is constant and, thus, $l_{t}$ is proportional ${ }_{488}$ to the total shielding area; the total shielding length $l_{t}$ is a helpful parameter 489 to avoid redundant material.

By varying the shielding length by steps of $20 \mathrm{~cm}$ in the range from $0 \mathrm{~cm}$ to $200 \mathrm{~cm}, 11^{6}$ configurations of module shields are explored. Fig. 11 shows the self-visibility $V_{s}$ as a function of the total shielding length $l_{t}$ for module shields with an ORC in place. Concerning $l_{t}=0$, approximately $8 \%$ of the backscattered neutrons hit the NEAT's detector area a second time if no shielding is installed, which can be decreased to about $2 \%$ by employing module shields. In accordance with the rather broad distribution, the configurations of smallest $V_{s}$ at a given $l_{t}$ are most efficient with regard to the $V_{s}-l_{t}$ ratio, and are referred to as 'low- $V_{s}$ configurations', and are indicated by the unfilled dots.

The inset of Fig. 11 shows the length of individual module shields in dependence on $l_{t}$ for the low- $V_{s}$ configurations, and reveals that alternating pairs of shields play a prominent role in efficiently reducing the cross-talk. While outer shields no. 1-3 and 10-12 remain relatively short in the range considered, one of the inner shield pairs exceeds the others. With regard to the configurations of increasing $l_{t}$, the prominent shielding pair increases continuously in length while its position shifts from the innermost pair no. 6, 7, through pair no. 5 , 8 , to, finally, pair no. 4,9 , which reaches a minimum self-visibility at a total shielding length of about $7.2 \mathrm{~m}$. A further increase in shielding lengths fails to significantly improve background suppression and, thus, the low- $V_{s}\left(l_{t}=7.2 \mathrm{~m}\right)$ module shield configuration is considered to be the optimum, and is shown in Fig. 2.

Apart from the layout with 12 module shields, we consider another layout with 415 equidistant shields, which are supposed to separate single detector tubes. Here, we limit the discussion to configurations in which all detector 


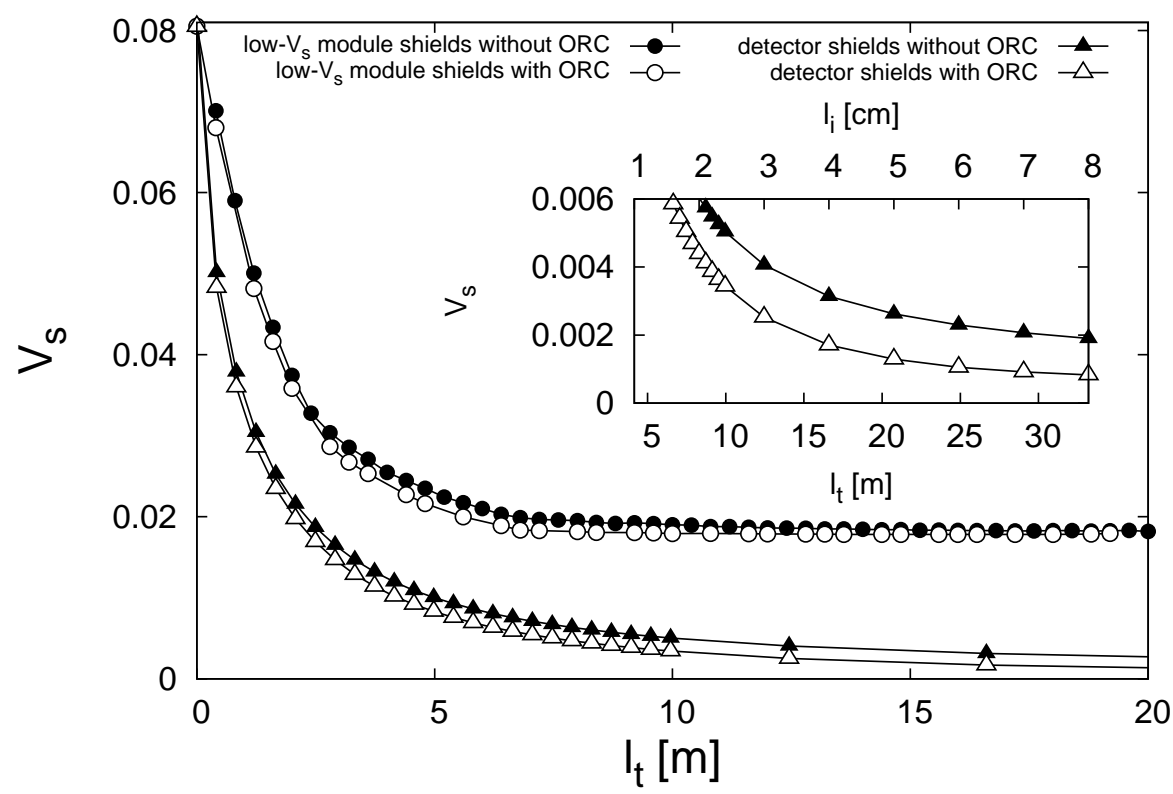

Figure 12: Self-visibility vs. $l_{t}$ for low- $V_{s}$ module shields (dots) and 415 equidistant detector shields (triangles). Filled symbols refer to setups without ORC, while unfilled symbols indicate setups with radial collimator in place. Data of low- $V_{s}$ module shields are taken from Fig. 11 and from a corresponding calculation without ORC which is not presented. Inset: $V_{s}$ as functions of $l_{t}$ (lower abscissa) and $l_{i}$ (upper abscissa) for 415 equidistant detector shields.

shields have the same length. Fig. 12 plots $V_{s}$ as a function of the total shielding length $l_{t}$ for this layout (415 detector shields), in comparison to that for the low$V_{s}$ module shields. The configurations of this layout are advantageous since they provide a smaller $V_{s}$ at a given $l_{t}$ than do the module shields, and realise smaller overall values of $V_{s}$ than those of the module shields.

The rather small cross-talk suppression through the use of ORC becomes a significant contribution at smaller background levels, as shown in the inset of Fig. 12. The reason for this becomes apparent from Fig. 13, which shows $V_{s}$ as a function of the time-of-flight for neutrons of $\lambda=5.1 \AA$ related to the moment of back-scattering. Regarding the detector system without shielding, $V_{s}$ decreases with the square of the length of the trajectory, and so with the timeof-flight, while there are more detectors at similar distances in the vicinity and in the opposite site of the cylindrical setup, resulting in a slight increase of $V_{s}$ 


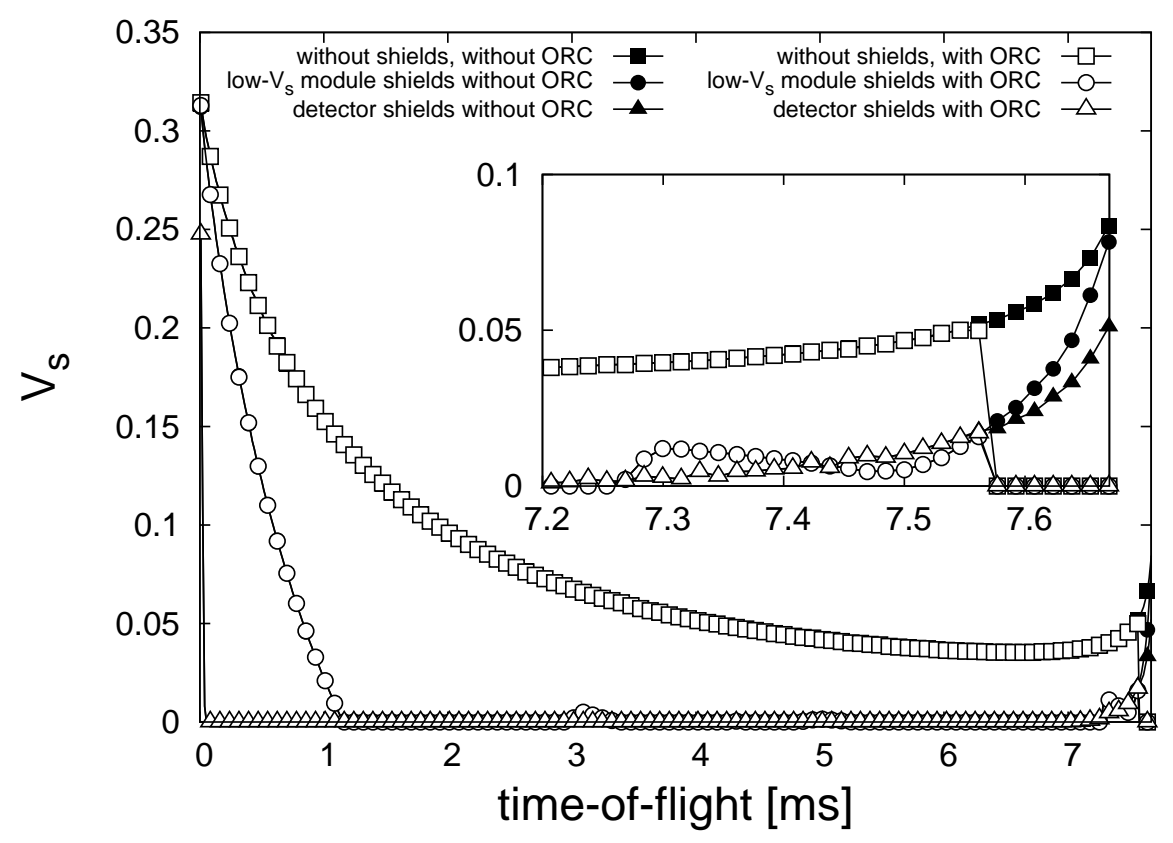

Figure 13: $V_{s}$ as a function of time-of-flight, assuming neutrons of wavelength $5.1 \AA$ for an instrument without shielding (squares), with low- $V_{s}\left(l_{t}=7.2 \mathrm{~m}\right)$ module shields derived from Fig. 11 (dots), and a layout of 415 detector shields of length $l_{i}=6 \mathrm{~cm}$ (triangles). Open symbols refer to setups with radial collimator while filled symbols denote setups without ORC. Inset: Details of back-scattered neutrons detected 7.2-7.7 ms after sample's signa

at larger time-of-flights. By introducing low- $V_{s}\left(l_{t}=7.2 \mathrm{~m}\right)$ module shields, the greater part of the intermediate and late time-of-flight background is prevented, while early cross-talk, occurring in-between the shields, is barely suppressed. In contrast, the use of 415 closely spaced detector shields of $6 \mathrm{~cm}$ length only permits back-scattering within the same tube and from opposing detectors, as indicated by the non-zero values around $0 \mathrm{~ms}$ and $7.6 \mathrm{~ms}$. The inset shows $V_{s}$ for late neutrons in more detail, and reveals that spurious scattering arriving within the last $0.1 \mathrm{~ms}$ is avoided by placing an ORC in the centre of symmetry. With 415 equidistant detector shields, late neutrons make up a significant part of the cross-talk, whose suppression decreases $V_{s}$ by about $50 \%$.

Regarding the setups without shielding and with 12 module shields of the low- $V_{s}\left(l_{t}=7.2 \mathrm{~m}\right)$ configuration, cross-talk vanishes with time-of-flight and, consequently, will mainly affect the energy loss tail of the sample's signal. With- 


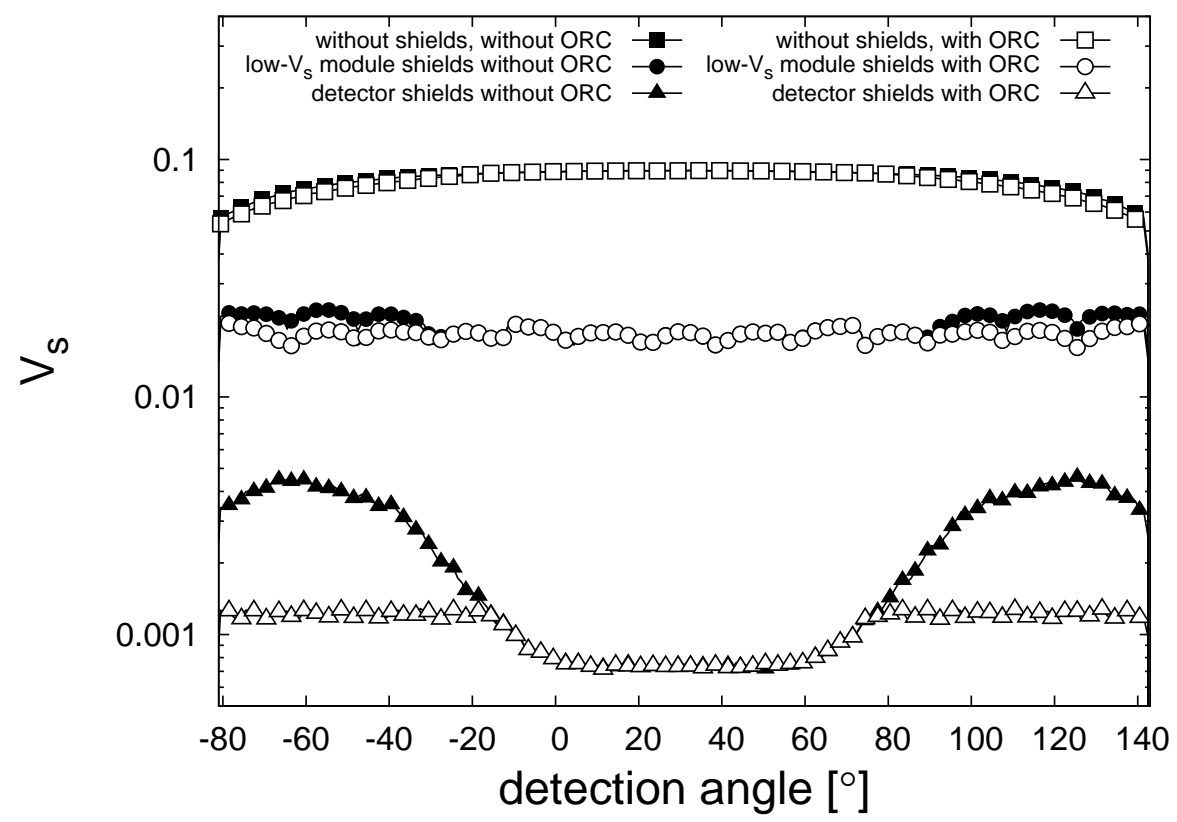

Figure 14: $V_{s}$ as a function of detection angle for instrument without shielding (squares), with low- $V_{s}\left(l_{t}=7.2 \mathrm{~m}\right)$ module shields (dots) derived from Fig. 11, and with 415 equidistant detector shields of length $l_{i}=6 \mathrm{~cm}$ (triangles). Open symbols refer to setups with radial collimator while filled symbols denote setups without ORC.

out shielding, the extended time-of-flight of the cross-talk lasts up to $7.7 \mathrm{~ms}$ and, thus, contributes to energy transfers of up to $5 \mathrm{meV}$. The introduction of the 12 low- $V_{s}\left(l_{t}=7.2 \mathrm{~m}\right)$ module shields narrows the concerned energies to $2 \mathrm{meV}$, still affecting so-called quasi-elastic studies which investigate the broadening of the elastic line. Compared with the other, more prominent source of background, the impact of spurious scattering from the sample environment is limited to the energy transfer range of up to $1 \mathrm{meV}$ wever, in contrast to the SE which is exposed to the incident beam and, thus, emits a constant amount of spurious scattering, detector cross-talk is proportional to the intensity at the detection

area. Consequently, detector cross-talk becomes an important contribution in

case of a scatterer with large scattering cross-section where one would expect a large signal-to-noise ratio from the SE. As a result, it is crucial for the quality of the data analysis to prevent the cross-talk between the detectors and its impact 
on a wide scope of energy transfers.

Fig. 14 shows $V_{s}$ as a function of the detection angle, taking into account that the centre of the detection system is rotated horizontally by $31^{\circ}$ relative to the incident beam. Without shielding, the centre of the detection system at $31^{\circ}$ has a maximum self-visibility, which decays as the detection angle changes, until it reaches a minimum at the edges $\phi_{1}=-83^{\circ}, \phi_{2}=143^{\circ}$. The introduction of low- $V_{s}\left(l_{t}=7.2 \mathrm{~m}\right)$ module shields suppresses the background considerably, while flattening the spectrum in spite of the 12 minima, which indicate the shield positions. With regard to the 415 equidistant detector shields of $6 \mathrm{~cm}$ length, cross-talk is further decreased, forming two levels of background. The larger level applies to the sides of the detection system, which are separated by a low-background region around the vertical symmetry axis at $31^{\circ}$. By placing an ORC, the background level at the sides is decreased, masking the direct line between opposing detector regions, while the low background in the centre is unaffected due to the absence of opposing detectors.

In contrast to the considered model, shielding is of finite thickness and requires a gap between the detectors, which can be an important aspect beside the self-visibility. On the one hand, gaps will be larger for the larger module shields compared to the smaller detector shields, since the thickness of shields increases with the individual shielding length to ensure mechanical stability. On the other hand, one may arrange the detector tubes as close as possible in the region between adjacent module shields in contrast to the setup with detector shields, which requires gaps between all detectors. As a result, the setup with module shields may be advantageous, e.g. in the case of a Bragg peak covering the area of two or more detectors, where gaps between all detectors would lead to a loss of detected intensity.

An additional consequence of the finite shielding thickness is the shadow effect, i.e. the shadow that a shield casts on the detectors. The width of the shadow increases with the thickness and the individual length of the shielding, i.e. to what extent the inner tip of the shielding approaches the sample. With regard to smaller shields of up to a few centimetres in length, the shadowed 
region is covered by the already neutron insensitive wall of the adjacent detector tubes that are located next to the shielding, and usually are about $0.5 \mathrm{~mm}$ thick. Moreover, if the detection angle covers both sides of the beam, one can offset the angular positions of the tubes at one side relative to the other to achieve an asymmetric arrangement of opposing tubes, as realised in NEAT. This arrangement would compensate for shadow effects or neutron insensitive regions and cover the Q-space continuously.

As a consequence of these considerations, NEAT relies on the setup of 415 equidistant detector shields with $l_{i}=6 \mathrm{~cm}$, whose length is rather an arbitrary choice based on cross-talk suppression, technical feasibility and handling of the detectors during the installation and maintenance. This shielding arrangement is expected to decrease the self-visibility of NEAT's detector system to approximately $0.1 \%$ if an ORC is in place and, thus, will reduce the cross-talk to about one-eightieth of its previous level.

\section{Summary}

In this paper, the suppression of spurious neutron scattering for the timeof-flight spectrometer NEAT was investigated. Two presumptive sources of background were identified, and two corresponding kinds of shielding have been studied. On the one hand, a radial collimator can be employed to mask the scattering from the sample environment, while, on the other hand, detector shielding has been designed to prevent the cross-talk of the radial detector system.

Although some approaches have already been applied to tailor a radial collimator to an instrument's needs, there has been a lack of a general concept to determine its parameters. Even if we assume infinitesimally thin blades, there remain three parameters which have to be defined: the inner radius, the outer radius, and the repeat angle. In some cases [19, 27], the maximum divergence $b_{0}$ of a neutron's trajectory that can pass the collimator vanes has served as a selection criterion parameter. However, there are many ways to realize the same value of $b_{0}$, such as, for instance, choosing long collimator vanes in combination with a large repeat angle, or smaller vanes with a smaller repeat angle. 
By slightly changing the analytical model of Copley et al. [27], it was shown that although radial collimators share the same $b_{0}$ and the same ability to suppress parasitic scattering from the sample environment, they differ in transmis-

sion, i.e. in the ability to let pass scattering from the sample. Since transmission

affects the measurement time directly, it is used as a second selection criterion to narrow the parameter space significantly. By considering the detection limit as a third criterion, which in fact can be expressed as a function of the former two, the three parameters of the ORC are optimized in the sense of SE scattering suppression, transmission, and the detection limit. However, an ORC's ability to suppress parasitic scattering as well as the resulting detection limit are sensitive to details of the sample environment used, limiting the optimization to NEAT's default setup.

Regarding the second main source of background, detector shielding was investigated for the suppression of the cross-talk between the detectors. By means of a Monte Carlo algorithm, the self-visibility of the detection area has been computed for two basic layouts of equidistant shields. The layouts differ in the number of shields: the first arrangement comprises 12 module shields, but the second consists of 415 detector shields. The configuration of 415 detector shields proves to be advantageous as it provides a lower level of cross-talk while using the same total amount of shielding material. Moreover, it has been found that a radial collimator prevents cross-talk between opposing detector parts, which are hard to suppress by detector shielding. As a result, an ORC's impact on the reduction of cross-talk becomes more significant the more efficient are the detector shields.

While this design of detector shielding is expected to apply to instruments of similar geometry, the optimization of the oscillating radial collimator is specific to the sample environment used. However, the fact that ORCs of comparable collimation differ in transmission is fundamental. 


\section{Acknowledgments}

We thank Ferenc Mezei for helpful comments and fruitful discussions.

[1] C. C. Lawrence, M. M. Flaska, M. Ojaruega, Andreas Enqvist, S. D. Clarke, S. A. Pozzi, and F. D. Becchetti. Time-of-flight measurement for energydependent intrinsic neutron detection efficiency. Nucl. Sci. Symp. Conf. Rec. (NSS/MIC), 2010 IEEE, pages 110-113, 2010.

[2] M. Kocsis, B. Farago, and M. Ceretti. New type of radial collimator for strain measurements by neutron diffraction. Rev. Sci. Instrum., 66:32-37, 1995.

[3] T. Pirling. A new high precision strain scanner at the ILL. Mater. Sci. Forum, 321-324:206-211, 2000.

[4] P. J. Withers, M. W. Johnson, and J. S. Wright. Neutron strain scanning using a radially collimated diffracted beam. Physica B, 292:273-285, 2000.

[5] D.-Q. Wang, X.-L. Wang, J. L. Robertson, and C. R. Hubbard. Modeling radial collimators for use in stress and texture measurements with neutron diffraction. J. Appl. Cryst., 33:334-337, 2000.

[6] S. Torii and A. Moriai. The design of the radial collimator for residual stress analysis diffractometer of J-PARC. Physica B, 385-386:1287-1289, 2006.

[7] A. F. Wright, M. Berneron, and S. P. Heathman. Radial collimator system for reducing background noise during neutron diffraction with area detectors. Nucl. Instr. Methods, 180:655-658, 1981.

[8] C. W. Tompson, D. F. R. Mildner, M. Mehregany, J. Sudol, R. Berliner, and W. B. Yelon. A position-sensitive detector for neutron powder diffraction. J. Appl. Cryst., 17:385-394, 1984. 
[9] J. Bouillot and J. Torregrossa. A radial oscillating collimator for small position sensitive detectors. Revue Phys. Appl., 19:799-800, 1984.

[10] J. Schefer, P. Fischer, H. Heer, A. Isacson, M. Koch, and R. Thut. A versatile double-axis multicounter neutron powder diffractometer. Nucl. Instr. and Meth. in Phys. Res. A, 288:477-485, 1990.

[11] E. Svab, G. Meszaros, and F. Deak. Neutron powder diffractometer at the budapest research reactor. Materials Science Forum, 228-231:247-252, 1996.

[12] A. Wannberg, A. Mellergård, P. Zetterström, R. Delaplane, M. Grönros, L.-E. Karlsson, and R. L. McGreevy. SLAD: A neutron diffractometer for the study of disordered materials. J. Neutron Res., 8:133-154, 1999.

[13] A. J. Studer, M. E. Hagen, and T. J. Noakes. Wombat: The high-intensity powder diffractometer at the OPAL reactor. Physica B, 385-386:1013-1015, 2006.

[14] N. Suzuki, M. Katano, M. Yonemura, and T. Kamiyama. Optimization of radial collimators for a powder diffractometer SPICA. JPS Conf. Proc., 8:036010, 2015.

[15] G. Ehlers, A. A. Podlesnyak, J. L. Niedziela, E. B. Iverson, and P. E. Sokol. The new cold neutron chopper spectrometer at the spallation neutron source: Design and performance. Rev. Sci. Instrum., 82:085108, 2011.

[16] J. R. D. Copley and J. C. Cook. The disc chopper spectrometer at NIST: a new instrument for quasielastic neutron scattering studies. Chem. Phys., 292:477-485, 2003.

[17] T. Unruh, J. Neuhaus, and W. Petry. The high-resolution time-of-flight spectrometer TOFTOF. Nucl. Instr. and Meth. in Phys. Res. A, 580:1414$1422,2007$. 
[18] D. Yu, R. Mole, T. Noakes, S. Kennedy, and R. Robinson. Pelican - a time of flight cold neutron polarization analysis spectrometer at OPAL. J. Phys. Soc. Jpn., 82(18):SA027, 2013.

[19] M. B. Stone, J. L. Niedziela, M. J. Loguillo, M. A. Overbay, and D. L. Abernathy. A radial collimator for a time-of-flight neutron spectrometer. Rev. Sci. Instrum., 85:085101, 2014.

[20] M. Nakamura, Y. Kawakita, W. Kambara, K. Aoyama, and R. Kajimoto et al. Oscillating radial collimators for the chopper spectrometers at MLF in J-PARC. JPS Conf. Proc., 8:036011, 2015.

[21] M. B. Stone, J. L. Niedziela, M. A. Overbay, and D. L. Abernathy. The ARCS radial collimator. EPJ Web of Conferences, 83:03014, 2015.

[22] R. I. Bewley, T. Guidi, and S. Bennington. MERLIN: a high count rate chopper spectrometer at ISIS. NNLS, 14(1):22-27, 2009.

[23] R. I. Bewley, J. W. Taylor, and S. M. Bennington. LET, a cold neutron multi-disk chopper spectrometer at ISIS. Nucl. Instr. and Meth. in Phys. Res. A, 637:128-134, 2011.

[24] K. Nakajima, S. Ohira-Kawamura, T. Kikuchi, M. Nakamura, and R. Kajimoto et al. AMATERAS: A cold-neutron disk chopper spectrometer. $J$. Phys. Soc. Jpn., 80:SB028, 2011.

[25] R. Kajimoto, M. Nakamura, Y. Inamura, K. Ikeuchi, and S. Ji et al. Recent improvement of the signal-to-noise ratio on the fermi chopper spectrometer 4SEASONS. J. Phys. Soc. Jpn., 82:SA032, 2013.

[26] J. Ollivier and H. Mutka. IN5 cold neutron time-of-flight spectrometer, prepared to tackle single crystal spectroscopy. J. Phys. Soc. Jpn., 80:SB003, 2011.

[27] J. R. D. Copley and J. C. Cook. An analysis of the effectiveness of oscillating radial collimators in neutron scattering applications. Nucl. Instr. and Meth. in Phys. Res. A, 345:313-323, 1994. 
[28] D. Wechsler, G. Zsigmond, F. Streffer, and F. Mezei. VITESS: Virtual instrumentation tool for pulsed and continuous sources. Neutron News, 11(4):25-28, 2000.

[29] K. Lieutenant, G. Zsigmond, S. Manoshin, M. Fromme, H. N. Bordallo, J. D. M. Champion, J. Peters, and F. Mezei. Neutron instrument simulation and optimization using the software package VITESS. Proc. of SPIE, 5536:134-145, 2004.

[30] P. A. Seeger, L. L. Daemen, E. Farhi, W.-T. Lee, L. Passell, J. Saroun, X.-L. Wang, and G. Zsigmond. Monte Carlo code comparisons for a model instrument. Neutron News, 13(4):24-29, 2002.

[31] G. Zsigmond, K. Lieutenant, S. Manoshin, H. N. Bordallo, J. D. M. Champion, J. Peters, J. M. Carpenter, and F. Mezei. A survey of simulations of complex neutronic systems by VITESS. Nucl. Instr. and Meth. in Phys. Res. A, 529:218-222, 2004.

[32] C. Zendler, K. Lieutenant, D. Nekrassov, and M. Fromme. VITESS 3 virtual instrumentation tool for the European Spallation Source. J. Phys.: Conf. Ser., 528:012036, 2014.

[33] F. Mezei and M. Russina. Neutron beam extraction and delivery at spallation neutron sources. Physica B, 283:318-322, 2000.

[34] Z. Izaola and M. Russina. Virtual design of the neutron guide for the TOF spectrometer NEAT. J. Phys.: Conf. Ser., 251(12064), 2009.

[35] Ed.: D. Reilly, N. Ensslin, and H. Smith. Passive Nondestructive Assay of Nuclear Materials. U.S. Nuclear Regulatory Research, Washington, 1991.

[36] T. C. Hansen, P. F. Henry, H. E. Fischer, J. Torregrossa, and P. Convert. The D20 instrument at the ILL: a versatile high-intensity two-axis neutron diffractometer. Meas. Sci. Technol., 19:034001, 2008. 
766 through $V_{W}$ ). 2012.

\section{AppendixA.}

with

$$
\text { t6r. }
$$

[37] N. Cuello and G. J. Cuello. Effects of the sample environment and collimation in the background measurement. J. Phys.: Conf. Ser., 340:012023,

[38] A. W. Hewat. Design for a conventional high-resolution neutron powder diffractometer. Nucl. Instr. and Meth., 127:361-370, 1975.

[39] D. A. Gedcke. How counting statistics controls detection limits and peak precision. Ortec Application Note, AN59, www.ortec-online.com/ServiceSupport/Library.aspx, accessed: 14. July 2015.

Consider a specific setup of the instrument with $W, r_{S E}=$ constant. In this case the denominator of Eq. (18) is constant and, thus, $G_{a n}\left(\phi_{D}\right)$ depends on $Q_{W}\left(\phi_{D}\right)$ of Eq. (16). The latter is defined by $V(r)$ and $V_{W}\left(r, \phi_{D}\right)$ which both involve an integration over $\psi$ with $t(b)$ as the integrand.

Assuming a negligible vane thickness of $\delta \equiv 0$ and, thus, $t_{0}=1, t(b)$ of Eq. (2) is a function of $b_{0}$. Consequently, $V(r)$ of Eq. (6) can be cast as [27]

$$
V(r)=\frac{2}{\pi}\left[\psi_{b}+\frac{r\left(\cos \psi_{b}-1\right)}{b_{0}}\right]
$$

while $V_{W}\left(r, \phi_{D}\right)$ of Eq. (10) may be written as [27]

$$
V_{W}\left(r, \phi_{D}\right)=\sum_{l=1}^{2} H\left(\psi_{l}^{+}-\psi_{l}^{-}\right)\left[f\left(\psi_{l}^{+}\right)-f\left(\psi_{l}^{-}\right)\right]
$$

$$
\begin{aligned}
f\left(\psi_{l}^{+/-}\right) & =\frac{1}{\pi} \int_{0}^{\psi_{l}^{+/-}} t(b) \mathrm{d} \psi, \\
& =\frac{1}{\pi}\left[\psi_{l}^{+/-}+\frac{r\left(\cos \psi_{l}^{+/-}-1\right)}{b_{0}}\right] .
\end{aligned}
$$

${ }_{764}$ Comparing Eqs. (A.1)-(A.3) reveals that the quotient of $V(r)$ and $V_{W}\left(r, \phi_{D}\right)$ and, thus, $G_{a n}\left(r, \phi_{D}\right)$ is a function of $b_{0}$ (apart from the dependence on $\phi_{D}$ 
The dependence of $V_{W}\left(r, \phi_{D}\right)$ on the detection angle $\phi_{D}$ is given through

$$
\frac{\partial f\left(\psi_{l}^{+/-}\right)}{\partial \phi_{D}}=\frac{1}{\pi}\left[\frac{\partial \psi_{l}^{+/-}}{\partial \phi_{D}}+\frac{r}{b_{0}} \frac{\partial\left(\cos \psi_{l}^{+/-}\right)}{\partial \phi_{D}}\right]
$$

768 where $\psi_{l}^{+/-}$is either $\pm \psi_{b}$ or of the form $a-\phi_{D}$ with $a= \pm \psi_{W}, \pi \pm \psi_{W}$; see Eq.

769 (12). The former value is a function of $b_{0}$ and is independent of $\phi_{D}$, while the

770 latter results in

$$
\frac{\partial f\left(\psi_{l}^{+/-}\right)}{\partial \phi_{D}}=\frac{a}{\pi}\left[1-\frac{r}{b_{0}} \sin \left(\phi_{D}-a\right)\right]
$$

771 where $b_{0}$ arises as a factor for the sinusoidal dependence on the detection angle.

772 However, even for collimator vanes of finite thickness the impact of $t_{0}$ on

773 Eqs. (A.1)-(A.5) is usually rather small compared to $b_{0}$ and, thus, the maxi-

774 mum impact parameter $b_{0}$ governs the figure of merit and its dependence on

775 the detection angle (e.g. shown in Fig. 7 for $\left\langle G_{m c}\right\rangle$ derived from the Vitess

776 calculations). 